\title{
Neural Pathways Underlying Lactate-Induced Panic
}

\author{
Philip L Johnson ',2, William A Truitt', Stephanie D Fitz', Christopher A Lowry² and Anantha Shekhar*,' \\ 'Department of Psychiatry and Pharmacology \& Toxicology, Indiana University School of Medicine, Indianapolis, IN, USA; ${ }^{2}$ Henry Wellcome \\ Laboratories for Integrative Neuroscience and Endocrinology, University of Bristol, Bristol, UK
}

\begin{abstract}
Panic disorder is a severe anxiety disorder characterized by susceptibility to induction of panic attacks by subthreshold interoceptive stimuli such as $0.5 \mathrm{M}$ sodium lactate infusions. Although studied for four decades, the mechanism of lactate sensitivity in panic disorder has not been understood. The dorsomedial hypothalamus/perifornical region (DMH/PeF) coordinates rapid mobilization of behavioral, autonomic, respiratory and endocrine responses to stress, and rats with disrupted GABA inhibition in the DMH/PeF exhibit panic-like responses to lactate, similar to panic disorder patients. Utilizing a variety of anatomical and pharmacological methods, we provide evidence that lactate, via osmosensitive periventricular pathways, activates neurons in the compromised DMH/PeF, which relays this signal to forebrain limbic structures such as the bed nucleus of the stria terminalis to mediate anxiety responses, and specific brainstem sympathetic and parasympathetic pathways to mediate the respiratory and cardiovascular components of the panic-like response. Acutely restoring local GABAergic tone in the DMH/PeF blocked lactate-induced panic-like responses. Autonomic panic-like responses appear to be a result of DMH/PeF-mediated mobilization of sympathetic responses (verified with atenolol) and resetting of the parasympathetically mediated baroreflex. Based on our findings, DMH/PeF efferent targets such as the $\mathrm{Cl}$ adrenergic neurons, paraventricular hypothalamus, and the central amygdala are implicated in sympathetic mobilization; the nucleus of the solitary tract is implicated in baroreflex resetting; and the parabrachial nucleus is implicated in respiratory responses. These results elucidate neural circuits underlying lactate-induced panic-like responses and the involvement of both sympathetic and parasympathetic systems. Neuropsychopharmacology (2008) 33, 2093-2107; doi: I0.1038/sj.npp. I301621; published online 5 December 2007
\end{abstract}

Keywords: panic; c-Fos; hypothalamus; GABA; anxiety; lactate

\section{INTRODUCTION}

A 'panic' response is a normal physiological survival reflex in humans (Stein and Bouwer, 1997) and can be elicited by either an exteroceptive- or interoceptive-cue perceived as life-threatening (Street et al, 1989; Ehlers and Breuer, 1996). Panic disorder is a severe anxiety disorder characterized by recurrent panic attacks, consisting of pronounced fear, as well as cardiovascular and respiratory responses (DSM-IV, 1994). The initial pathology in these patients appears to be an alteration somewhere in the central neural pathways regulating normal panic responses, thus rendering the patients susceptible to unprovoked panic symptoms when exposed to ordinarily mild interoceptive stressors (Vickers and McNally, 2005). This is supported by findings that panic disorder patients, but not healthy controls, are hyperresponsive to normal interoceptive cues (Hoehn-Saric et al, 2004; Pollock et al, 2006), and are also susceptible to induction of panic attacks by interoceptive stimuli such as

*Correspondence: Dr A Shekhar, Department of Psychiatry, Indiana University School of Medicine, I I I I W. I Oth, PB 308, Indianapolis, IN 46202, USA, Tel: I 317274 1246, Fax: I 317278 482।,

E-mail: ashekhar@iupui.edu

Received 3 April 2007; revised 5 October 2007; accepted 6 October 2007 intravenous (i.v.) $0.5 \mathrm{M}$ sodium lactate infusions and $7.5 \%$ $\mathrm{CO}_{2}$ inhalations (Liebowitz et al, 1986; Gorman et al, 1994). In light of this, these specific interoceptive challenges that fail to induce panic-like responses in healthy controls are often referred to as 'subthreshold' interoceptive challenges.

Removal of local tonic $\gamma$-amino butyric acid (GABA)mediated inhibition in the dorsomedial hypothalamic/ perifornical region $(\mathrm{DMH} / \mathrm{PeF})$ in rats results in rapid mobilization of a response characterized by behavioral, autonomic, respiratory, and endocrine components of panic-like responses (see review (DiMicco et al, 2002)). In light of this, Shekhar and colleagues developed an animal model of panic disorder where chronically inhibiting GABA synthesis in the $\mathrm{DMH} / \mathrm{PeF}$ of rats leads to heightened anxiety and panic-like responses following exposure to i.v. infusions of $0.5 \mathrm{M}$ sodium lactate (Shekhar et al, 1996; Shekhar and Keim, 1997; Johnson and Shekhar, 2006) or 7.5\% $\mathrm{CO}_{2}$ inhalation (Fitz et al, 2003).

Although 'osmosensitive' circumventricular organs (CVOs) such as the organum vasculosum of the lamina terminalis (OVLT) appear to be critical for relaying the lactate signal to the DMH/PeF (Johnson and Gross, 1993; Shekhar and Keim, 1997; Thompson and Swanson, 1998), the specific regions of the $\mathrm{DMH} / \mathrm{PeF}$ involved and the downstream neural pathways through which this signal is propagated to behavioral centers to induce anxiety and 
elicit increases in heart rate (HR), mean arterial blood pressure (MAP) and respiration rate (RR) are poorly understood. Since evidence from previous studies suggested that disinhibiting the DMH elicits cardioexcitatory responses by mobilizing sympathetic outflow (see review (DiMicco et al, 2002)) and resetting the sensitivity of the baroreflex, which is parasympathetically mediated (see review McDowall et al, 2006), we hypothesized that disinhibition of the $\mathrm{DMH} / \mathrm{PeF}$ alters lactate-induced behavioral, respiratory, and cardiovascular responses through efferent targets involved in central sympathetic and parasympathetic autonomic control (Dampney, 1994; Thompson and Swanson, 1998; Fontes et al, 2001).

To identify neural pathways involved in sodium lactateinduced panic-like responses, including specific regions of the $\mathrm{DMH} / \mathrm{PeF}$, we studied the effects of sodium lactate or vehicle infusions in rats with prior infusions of the GABA synthesis inhibitor L-allylglycine (L-AG; 2-amino-4-pentenoic acid, an inhibitor of glutamic acid decarboxylase), or the inactive isomer D-allylglycine (D-AG: controls), into the $\mathrm{DMH} / \mathrm{PeF}$. We then utilized a variety of immunohistochemical techniques to investigate immediate-early gene (c-Fos) induction in neurochemically and anatomically identified neuronal populations. We also conducted site-specific pharmacological interventions to confirm the involvement of these pathways.

\section{MATERIALS AND METHODS}

\section{Animals}

Adult male Sprague-Dawley rats (300-350 g; Harlan Laboratories, Indianapolis, IN) were used in all experiments and were housed individually in plastic cages under standard environmental conditions $\left(22^{\circ} \mathrm{C} ; 12 / 12\right.$ light/dark cycle; lights on at 0700 hours) for 7-10 days prior to the surgical manipulations. Food and water were provided ad libitum. Animal care procedures were conducted in accordance with the NIH Guidelines for the Care and Use of Laboratory Animals (NIH Publication no. 80-23) revised 1996 and the guidelines of the IUPUI Institutional Animal Care and Use Committee.

\section{Surgical Procedures}

For experiments 1 and 4, rats were anesthetized with an isoflurane system (MGX Research Machine; Vetamic, Rossville, IN). MAP and HR were measured by a femoral arterial line connected to a telemetric probe which contained a pressure transducer (Cat. no. C50-PXT, Data Science International (DSI) St. Paul, MN) and monitored by DSI DATAQUEST software. For experiments 2 and 3, MAP, HR, and RR were monitored by a femoral arterial line attached to a pressure transducer connected to a Beckman R511 Dynograph (Beckman Instruments Inc., Fullerton, CA). In experiments 1 and 4, the HR and MAP data are changes in HR and MAP from the average of the baseline ( $t-5$ to $t-1)$ from each rat over $20 \mathrm{~min}$. In experiments 2 and 3, the data are expressed as peak changes in HR or MAP, which was defined as the highest value sustained for over $1 \mathrm{~min}$. All rats were fitted with venous catheters for i.v. infusions.
After 3 days of recovery, rats were tested for baseline cardiovascular and respiratory responses to lactate, then anesthetized and T-shaped 26-gauge cannulae (Cat. no. 3260PG, Plastics One, Ranoake, VA) were directed at cardioexcitatory regions of the DMH (Samuels et al, 2004) based on the following stereotaxic coordinates relative to bregma (using a $10^{\circ}$ angle from the vertical plane with the incisor bar set at $+5 \mathrm{~mm}$ were: anterior $-1.2 \mathrm{~mm}$, lateral + $2.5 \mathrm{~mm}$ and ventral $+7.0 \mathrm{~mm}$. A total of 50 pmol per $100 \mathrm{nl}$ of bicuculline methiodide (BMI), a $\mathrm{GABA}_{\mathrm{A}}$ receptor antagonist, was injected through the vertical arm of the cannula to ascertain that the tip of the cannula was placed in a cardioexcitatory region (ie where BMI elicits $\geqslant 50$ beats per min in HR). This microinjection commenced following an $\sim 10 \mathrm{~min}$ stable baseline in HR and MAP. The cannulae were then retracted and filled with either L- or D-AG solution, then redirected to the previous coordinates. The osmotic minipumps (DURECT Corp., Cat. no. 2002) were then attached to the cannula with PE-60 tubing and sutured into place subcutaneously at the nape of the neck. The concentration of the solutions in the minipumps was such that $3.5 \mathrm{nmol}$ per $0.5 \mu \mathrm{l}$ per hour of $\mathrm{L}-$ or $\mathrm{D}-\mathrm{AG}$ was infused into the $\mathrm{DMH}$ region over 5 days prior to i.v. infusions. Previous studies have determined that the dose of $\mathrm{L}-\mathrm{AG}$ utilized here reduces local GABA concentrations by approximately $60 \%$ following unilateral infusions (based on microdialysis study (Abshire et al, 1988), and enzyme assays (Shekhar and DiMicco, 1987; Shekhar et al, 1996; Shekhar and Keim, 1997; Shekhar et al, 2006), and supported by immunohistochemistry (Johnson and Shekhar, 2006), and increases anxiety-like behavior (ie as measured by the social interaction (SI) test, and elevated plus-maze (EPM)) without increasing cardiorespiratory responses (Shekhar and DiMicco, 1987; Shekhar et al, 1996; Shekhar and Keim, 1997; Shekhar et al, 2006).

\section{Sodium Lactate Infusion}

A total of 5 days following stereotaxic surgery, rats randomly received i.v. infusions of $0.5 \mathrm{M}$ sodium lactate or $0.9 \%$ saline over $15 \mathrm{~min}$, similar to clinical lactate infusions (Liebowitz et al, 1986). Cardiorespiratory responses were recorded continuously for $5 \mathrm{~min}$ prior to infusion (baseline) until $15 \mathrm{~min}$ following the onset of sodium lactate infusion.

\section{Social Interaction Test}

The SI test was utilized to measure anxiety-like behavior. This test was chosen for the following reasons: (1) the SI test is a fully validated test of experimental anxiety-like behavior in rats (File, 1980) and (2) the dose of L-AG used here increases anxiety-like behavior as measured by the SI test and also other measures of anxiety such as the EPM (Shekhar et al, 1996). Consequently, the SI test may be a sensitive measure of an increase in anxiety-like state following intra-DMH infusions of $\mathrm{L}-\mathrm{AG}$. The SI test procedure as used in our laboratory has been described previously (Shekhar and Katner, 1995; Johnson and Shekhar, 2006). See also Supplementary methods for detailed description. 
Experiment 1: cellular responses in $L-A G$ or $D$-AG-treated rats following lactate or saline challenge. Results from experiment 1 are derived from the same rats that were used in a previous report (Johnson and Shekhar, 2006). We have previously reported maximum changes in MAP and HR following vehicle or sodium lactate infusion in these L-AGand D-AG-treated rats (Johnson and Shekhar, 2006) but here we describe the detailed time course of these responses. We have also previously reported effects of treatment on anxiety-related behavior in the SI test (Johnson and Shekhar, 2006). Here, we describe changes in RR which have not been reported previously. Here, we also describe correlations between MAP, HR, RR, and behavioral scores in the SI test and c-Fos expression within specific brain regions in order to gain insight into the potential pathways mediating these responses. Finally, we have previously reported data from series of brain sections of the $\mathrm{DMH} / \mathrm{PeF}$ region stained or immunostained with (1) methyl green, (2) GABA, (3) c-Fos combined with the NR1 subunit of the $N$-methyl-D-aspartate (NMDA) receptor, and (4) c-Fos combined with the GluR2, 3 subunit of the alpha-amino3-hydroxy-5-methyl-4-isoxazolepropionate (AMPA) receptor. Here, we examine c-Fos expression, in combination with specific neurochemical markers, in the efferent pathways of the $\mathrm{DMH} / \mathrm{PeF}$ region that are believed to be responsible for the autonomic and behavioral responses to sodium lactate in intra-DMH/PeF L-AG-treated rats.

\section{Perfusion}

Methods for perfusion and for verification of cannulae placements in experiment 1 have been described previously (Johnson and Shekhar, 2006). Briefly, L-AG and D-AG rats received i.v. infusions of either lactate or saline vehicle over $15 \mathrm{~min}$ ( $n=6 /$ group) were immediately tested in the SI test, then anesthetized and perfused with a $4 \%$ paraformaldehyde per $1.5 \%$ sucrose solution and processed for immunohistochemistry $90 \mathrm{~min}$ following the onset of the i.v. infusions as described previously (Johnson and Shekhar, 2006). In the present study, four of the six alternate sets of $30 \mu \mathrm{m}$ coronal brain sections were stained, one set each for c-Fos (full brain), c-Fos/tyrosine hydroxylase (TH, brainstem only), c-Fos/choline acetyltransferase (ChAT, medulla only), c-Fos/GAD67 (medulla only) and c-Fos/neuronal marker $n$ (NeuN, midbrain only)). Immunostaining of $\mathrm{TH}$, ChAT and GAD67 was done to identify catecholaminergic (ie norepinephrine- and epinephrine-synthesizing), acetycholinergic and GABAergic neurons, respectively. Noradrenergic (norepinephrine-synthesizing) and adrenergic (epinephrine-synthesizing) cell groups in the brainstem were defined according to a standard stereotaxic atlas of the rat brain (ie (Paxinos and Watson, 1997)) as described previously (Johnson et al, 2005).

\section{Single/double Immunohistochemistry}

We used immunohistochemical staining for c-Fos, in the presence or absence of other neurochemical markers, to identify specific brain regions involved in responses to $\mathrm{L}-\mathrm{AG}$ and sodium lactate. Measurement of c-Fos expression is a useful method of identifying functional cellular responses among large populations of neurons in the CNS. Despite some limitations, for example, (1) a lack of cellular c-Fos induction does not necessarily imply a lack of neuronal responses, and (2) measurement of c-Fos expression is not likely to reflect different levels of increased neuronal activity or subtle differences in the patterns of neuronal firing, this technique has been a useful tool in the functional mapping of neural circuitry mediating or modulating many stimuli, including stress-related stimuli (reviewed in Herman et al, 1996; Herman and Cullinan, 1997; Senba and Ueyama, 1997; Kovacs, 1998).

Due to the detailed rostrocaudal analyses of c-Fos double immunostaining, chromogens were utilized in place of immunofluorescence. Although fluorescence immunostaining is critical when co-localizing two or more cytoplasmic or nuclear substrates, two chemical substrates can be co-localized using chromogen reactions when one is nuclear and the other cytoplasmic or membrane associated. The use of chromogens, vs fluorescence, also allowed detailed and repeated analyses of archived slides and does not suffer from a reduced signal following routine imaging as is the case with fluorescence. Furthermore, white matter fiber tracts, which serve as anatomical landmarks for identification of the specific rostrocaudal levels for analysis, are easier to locate using sections with chromogen-based immunostaining. Immunostaining for c-Fos protein was accomplished using primary antiserum directed against c-Fos (rabbit anti-c-Fos-polyclonal, affinity-purified antibody, Cat. no. PC38, Ab-5, Calbiochem, San Diego, CA, USA; diluted 1:10000) as described previously (Johnson et al, 2005). The chromogen for immunostaining of c-Fos was SG (Cat. no. SK-4700, Vector Laboratories, Burlingame, CA, USA) and the reaction was run for $20 \mathrm{~min}$.

For double immunostaining, immunostaining of c-Fos protein was done first and then, after the first chromogen reaction had been quenched using $1 \% \mathrm{H}_{2} \mathrm{O}_{2}$, immunostaining of $\mathrm{TH}, \mathrm{ChAT}, \mathrm{GAD} 67$, or NeuN protein immunostaining was done second using primary antibodies/antiserum directed against c-Fos (as above) then TH (rabbit antiTH-polyclonal, affinity-purified antibody, Cat no. B1562, Chemicon, Temecula, CA, USA; diluted 1:10000), ChAT (sheep anti-ChAT polyclonal, antiserum, Cat. no. AB1582, Chemicon, diluted 1:5000), GAD67 (mouse anti-GAD67, monoclonal affinity-purified antibody, Cat. no. AB5406, Chemicon, diluted 1:1500), or NeuN (mouse anti-NeuN monoclonal affinity-purified antibody, Cat. no. MAB377, Chemicon, diluted $1: 10000$ ) using standard immunohistochemical methods as described previously (Johnson et al, 2005) and as described in Supplementary methods.

\section{Data Generation from Immunohistochemistry}

Brain regions analyzed for the number of c-Fos-ir nuclei were chosen based on previous studies implicating them in fear circuitry (Singewald and Sharp, 2000; Singewald et al, 2003) and central autonomic and/or osmotic regulation (see reviews Johnson and Gross, 1993; Dampney, 1994). Cell counts for each brain region are based on cell counts from a single brain section from each rat from each treatment group. Each brain section was selected to represent the specific rostrocaudal level, with reference to distance from bregma using a standard stereotaxic atlas of the adult rat brain (Paxinos and Watson, 1997) (see also Supplementary 
Figure S1). In cases, where a specific brain region is represented bilaterally, the cell counts for the left and right sides were pooled for a single measurement. Details on data generation from single and double IHC are available in the Supplementary methods.

\section{Statistical Analyses}

Each dependent variable for in vivo analyses (ie SI duration, HR, MAP, RR) was analyzed using a two-way ANOVA with repeated measures with lactate and isomer as main factors and time as repeated measures. In the presence of significant main effects or main effect $\times$ time interactions, post hoc tests were conducted to define the anatomical location of the effects using Tukey's HSD tests which corrects for multiple comparisons.

Because we have analyzed a large number of brain regions, we have taken several steps to minimize type I errors (ie detecting a difference when no difference exists). Data for each dependent variable (c-Fos, c-Fos/TH, TH, c-Fos/ChAT, ChAT, c-Fos/NeuN, and NeuN) were analyzed using a separate two-way ANOVA with repeated measures with lactate and isomer as main factors and brain region as a repeated measure. In the presence of significant main effects or main effect $\times$ brain region interactions in the overall model, secondary two-way ANOVAs with lactate and isomer as main factors were conducted for each brain region counted. In the presence of appropriate and significant main effects or interactions in both the overall and secondary ANOVAs, post hoc pairwise comparisons were conducted to define the anatomical location of the effects using Tukey's HSD tests.

Since the full model in the two-way ANOVA with repeated measures cannot be estimated if there are missing values, missing values were handled according to the methods of Petersen (1985). Calculated missing values were only used to conduct the overall multifactor ANOVA, and were not used for subsequent secondary ANOVAs, pairwise comparisons or in the report of the data. Details of missing values are described in Supplementary methods.

All statistical analyses were carried out using SYSTAT 5.02 (SYSTAT Inc., San Jose, CA) and SPSS 14.0 (SPSS Inc., Chicago, IL), and all graphs were generated using SigmaPlot 2001 (SPSS Inc.). Correlations were done for the number of c-Fos-ir cells and the SI duration or autonomic responses in all analyses. Only significant correlations are mentioned in the results.

Experiment 2: muscimol into the $D M H / P e F$ of $L-A G+$ lactate-treated rats. All rats $(n=6)$ were treated with $\mathrm{L}-\mathrm{AG}$ as previously described and received unilateral injections of 100 pmol muscimol, a $\mathrm{GABA}_{\mathrm{A}}$ receptor agonist, in aCSF, or $100 \mathrm{nl}$ of aCSF alone (in random order) into the $\mathrm{DMH} / \mathrm{PeF}$ (using a 33-gauge injector (Cat. no. C315I, Plastics One), which extended $1 \mathrm{~mm}$ past the 24-gauge cannula (Cat. no. C315G, Plastics One)) $30 \mathrm{~min}$ prior to $0.5 \mathrm{M}$ sodium lactate infusions. A total of $48 \mathrm{~h}$ was allowed between randomized injections.

Experiment 3: muscimol into the BNST of $L-A G+$ lactatetreated rats. All rats $(n=5)$ were treated with $\mathrm{L}-\mathrm{AG}$ into the $\mathrm{DMH} / \mathrm{PeF}$ as previously described and received unilateral injections of muscimol or saline directed at the BNST $30 \mathrm{~min}$ prior to receiving i.v. infusions of $0.5 \mathrm{M}$ sodium lactate. Stereotaxic coordinates relative to bregma for the BNST, using a $10^{\circ}$ angle from the vertical plane with the incisor bar set at $+5 \mathrm{~mm}$ were: anterior $+1.0 \mathrm{~mm}$, lateral + $2.5 \mathrm{~mm}$ and ventral $-7.0 \mathrm{~mm}$. On post-pump day 5 , L-AGtreated rats were injected, in random order, with either aCSF $(100 \mathrm{nl})$ or muscimol $(250 \mathrm{pmol}$ per $100 \mathrm{nl})$ directly into the BSNT (using a 33-gauge injector (Cat. no. C315I, Plastics One), which extended $1 \mathrm{~mm}$ past the 24-gauge cannula (Cat. no. C315G, Plastics One)). A total of $48 \mathrm{~h}$ was allowed between randomized injections.

Experiment 4: intraperitoneal atenolol in $L-A G+$ lactatetreated rats. In order to confirm peripheral sympathetic contributions to panic-like cardiovascular responses, all rats $(n=5)$ were treated with $\mathrm{L}-\mathrm{AG}$ into the $\mathrm{DMH} / \mathrm{PeF}$ as previously described and received intraperitoneal (i.p.) injections of $10 \mathrm{mg} / \mathrm{kg}$ atenolol $30 \mathrm{~min}$ prior to receiving i.v. infusions of $0.5 \mathrm{M}$ sodium lactate. Cardiovascular data were obtained as previously described in the Materials and Methods section after rats received (1) L-AG + i.v. lactate, (2) $\mathrm{L}-\mathrm{AG}+$ i.p. atenolol + i.v. saline or (3) $\mathrm{L}-\mathrm{AG}+$ i.p. atenolol + i.v. lactate. A total of $48 \mathrm{~h}$ was allowed between each of the randomized infusions of saline + atenolol or lactate + atenolol or lactate alone.

\section{RESULTS}

\section{Experiment 1: Cellular Responses in L- or D-AG Rats Following Lactate or Saline Challenge}

Behavioral and cardio-respiratory responses in $L-A G$ or $D$-AG-treated rats following i.v. challenges. Part of the physiological responses (maximal changes in HR and MAP following i.v. saline or sodium lactate infusions, before and after intra-DMH L-AG or D-AG infusions) from experiment 1 were published previously (Johnson and Shekhar, 2006). In the present article, we wanted to determine the onset and duration of the cardiorespiratory responses following the sodium lactate infusions. We also present for the first time the SI test data and the RR data, which we were able to obtain as an indirect measurement following analysis of normal sinus arrhythmia. Rats with L-AG infusions into the $\mathrm{DMH} / \mathrm{PeF}$ region for 5 days had less SI (an anxiogenic effect) in the SI test measured immediately after a $20 \mathrm{~min}$ i.v. infusion of either vehicle or sodium lactate compared to pre-pump baseline behavior and to D-AG control rats ((Figure 1a), lactate $\times$ isomer $\times$ time interaction $\left(\mathrm{F}_{(1,18)}=\right.$ 9.7, $p=0.006)$ ). There were no significant differences in baseline SI times among treatment groups prior to either D- or L-AG infusions.

Between-subjects analyses revealed that rats with intra$\mathrm{DMH} / \mathrm{PeF}$ L-AG infusions, but not D-AG controls, responded to lactate infusions with hypertension (Figure $1 \mathrm{~b}$; lactate $\times$ isomer $\times$ time interaction $\left.\left(\mathrm{F}_{(192,285)}=4.1, p<0.001\right)\right)$, tachypnea (Figure 1c; lactate effect $\left.\left(\mathrm{F}_{(3,16)}=4.0, p<0.05\right)\right)$ and tachycardia (Figure 1d; lactate $\times$ isomer $\times$ time interaction $\left.\left(\mathrm{F}_{(12,285)}=2.6, p<0.001\right)\right)$. Within-subjects analyses revealed that that only $\mathrm{L}-\mathrm{AG}+$ lactate-treated rats had an increase in MAP (time effect, $\mathrm{F}_{(19,80)}=4.7, p<0.001$ ), and $\mathrm{HR}$ (time effect, $\mathrm{F}_{(19,80)}=1.7, p<0.05$ ) from baseline and an 
a

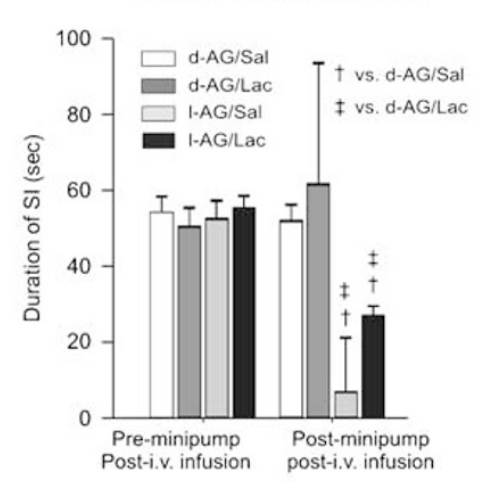

C

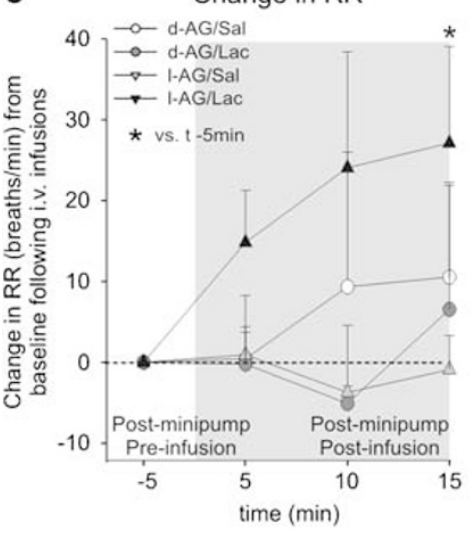

b

Change in MAP

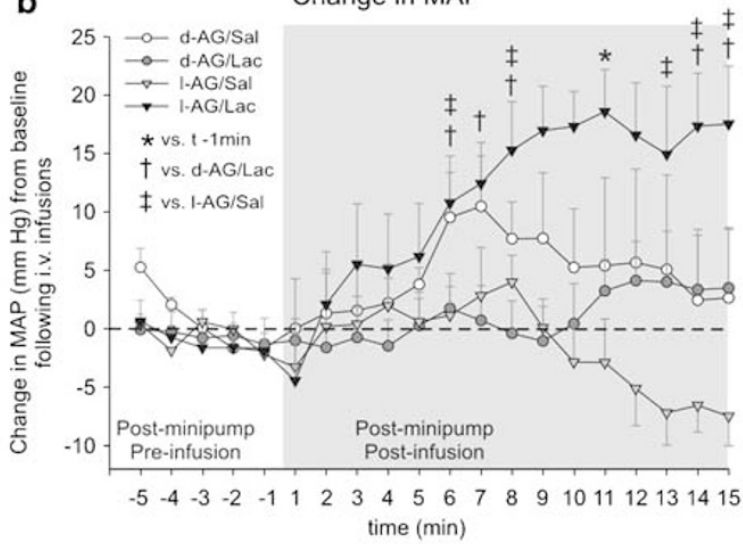

d

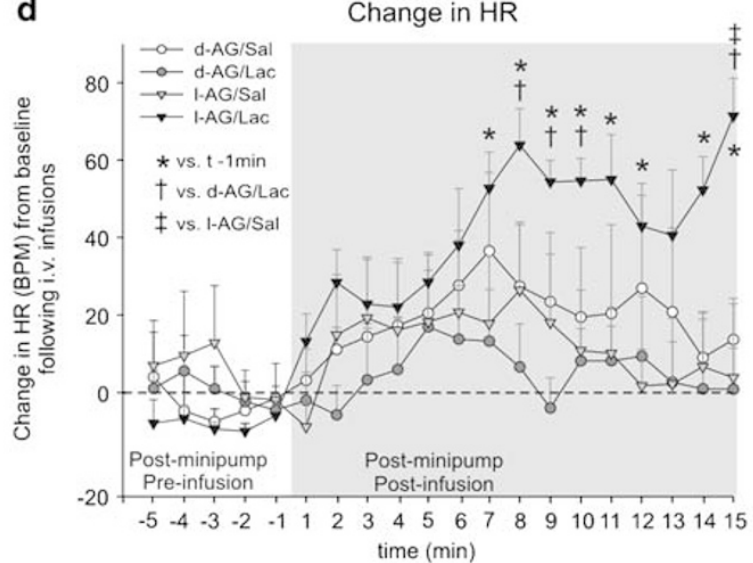

Figure I The effect of GABA synthesis inhibition in the DMH region and i.v. lactate infusions on anxiety-like behavioral and cardiorespiratory responses from experiment I. (a) Social interaction (SI) time pre- and post-L-AG (GABA synthesis inhibitor)- or D-AG (control isomer) treatment in rats also receiving i.v. infusions of saline (Sal) or lactate (Lac). Change in (b) mean arterial blood pressure (MAP), (c) respiration rate (RR), (d) and heart rate (HR) from 5 min baseline post-i.v. infusion. The RR data are presented as change from baseline for consistency, but the $\left(^{*}\right)$ symbol indicates a significant difference in actual RR over time. Bars or lines represent the mean + SEM. No differences were noted between baseline levels of MAP, RR, and HR for each group (data not shown).

increase in RR (time effect, $\mathrm{F}_{(3,16)}=4.0, p<0.05$ ) over time following i.v. infusions. There were no differences in baseline HR, MAP, or RR among treatment groups prior to L-AG or after D-AG treatment (data not shown) and there was no change in the duration of SI (Figure 1a) or cardiorespiratory arousal following lactate infusions in D-AG control rats (Figure $1 \mathrm{~b}-\mathrm{d}$ ).

All minipump cannulae placements resided in regions of the $\mathrm{DMH} / \mathrm{PeF}$ and posterior hypothalamus known to be cardioexcitatory and also verified for each infusion site in the present study to be cardioexcitatory (see the Materials and Methods section). An illustration of the minipump placements for experiment 1 has been previously published (Johnson and Shekhar, 2006). Some tissue damage at the site of implantation was noted due to the 26-gauge cannula $(0.46 \mathrm{~mm}$ outside diameter), but this damage was equal in the control and experimental groups (data not shown). Previous analyses of the total numbers of NMDA and AMPA receptor-ir neurons in the $\mathrm{DMH}$ did not differ between the side of the DMH which had the cannula track compared to the intact $\mathrm{DMH}$ on the contralateral side (Johnson and Shekhar, 2006). Overall this suggests that the tissue damage within the DMH was minimal. Therefore, any treatment effects are unlikely to be due to mechanical damage of the DMH due to the cannula.

Overall analyses of $c$-fos cell counts following single immunostaining in experiment 1 . Brain regions analyzed for the number of c-Fos-ir nuclei were chosen based on previous studies implicating them in fear circuitry (Singewald and Sharp, 2000; Singewald et al, 2003) and central autonomic and/or osmotic regulation (see reviews (Johnson and Gross, 1993; Dampney, 1994)). Analysis of the number of c-Fos-ir nuclei in all brain regions analyzed using two-factor ANOVA with repeated measures revealed a lactate $\left(\mathrm{F}_{(1,19)}=12.0, p=0.003\right)$ and isomer $\left(\mathrm{F}_{(1,19)}=4.8\right.$, $p=0.041)$ effect, but no lactate $\times$ isomer interaction $\left(\mathrm{F}_{(1,19)}=1.2, p=0.279\right)$. Additionally, the effects of both lactate and isomer treatment on the number of c-Fos-ir cells were dependent on brain region (lactate $\times$ brain region interaction $\left(\mathrm{F}_{(66,1254)}=6.9, p<0.001\right)$; isomer $\times$ brain region interaction $\left.\left(\mathrm{F}_{(66,1254)}=3.3, p<0.001\right)\right)$. Furthermore, a lactate $\times$ isomer $\times$ brain region interaction was observed $\left(\left(\mathrm{F}_{(66,1254)}=2.7, p<0.001\right)\right)$. Overall, L-AG-treated rats, but not D-AG-treated rats, had altered c-Fos responses in some 
brain regions, but not others, in response to sodium lactate infusions. Detailed descriptions of these effects within each brain region are discussed below. Secondary two-way ANOVAs for each brain region with lactate and isomer as main factors are listed in Supplementary Table S1a-d.

\section{Circumventricular organs implicated in 'sensing' sodium lactate.}

Experiment 1: Lactate infusions increased the number of c-Fos-ir cells in the OVLT (Figure 2a-b), subfornical organ (SFO) and area postrema (AP) in both L-AG- and D-AGtreated rats (Table 1). However, as predicted, c-Fos responses in the OVLT, SFO, and AP did not correlate with SI duration or cardiorespiratory responses (see Supplementary results for details).

c-Fos responses in CNS regions implicated in regulating anxiety-like behavior.

Rostral BNST/LSV and BLA: Infusing L-AG in the DMH/ $\mathrm{PeF}$, regardless of lactate challenge, increased the number of c-Fos-ir cells in the medial BNST (BSTM) and the ventral part of the lateral septum (LSV), but not in the rostral part of the lateral BNST (BSTL) following exposure to the SI test, compared to D-AG-treated rats (Table 1). Furthermore, analysis of data from all rats in the study revealed that c-Fos responses in the BSTM were inversely correlated with the change in the duration of SI from pre-intra-DMH/PeF to post-intra-DMH/PeF infusions (see Supplementary results for details).

Cellular c-Fos responses in the caudal basolateral amygdala (BLA) were similar to the patterns observed in the BSTM. Here, L-AG treatment and exposure to the SI test, irrespective of lactate challenge, increased the number of c-Fos-ir cells (Table 1). The number of c-Fos-ir cells in the BLA negatively correlated with the duration of SI from preintra-DMH/PeF infusions to post-intra-DMH/PeF infusions $\left(r^{2}=0.20, p=0.040\right)$.
c-Fos responses in CNS regions implicated in autonomicl respiratory regulation.

Caudal BNST/LSV and CeA: A greater number of c-Fosir cells were observed in the caudal BSTL and LSV of L-AG + lactate-treated rats compared to other groups (Table 1). In addition, c-Fos responses in the caudal BSTL were positively correlated with the mean change in HR over the 15 min following i.v. infusions $\left(r^{2}=0.33, p=0.012\right)$.

In contrast to the BLA, lactate increased the number of c-Fos-ir cells in the rostral capsular, lateral, and medial parts of the central amygdala $(\mathrm{CeC}, \mathrm{CeL}$, and $\mathrm{CeM}$, respectively; Table 1) and in the caudal $\mathrm{CeC}$ and $\mathrm{CeL}$ (Supplementary Table S2, Supplementary Figure S2a-b) in $\mathrm{L}-\mathrm{AG}$, not D-AG, -treated rats. In addition, $\mathrm{c}$-Fos responses in the rostral CeM $\left(r^{2}=0.27, p=0.023\right)$ and caudal $\mathrm{CeC}$ $\left(r^{2}=0.30, p=0.016\right)$ and $\mathrm{CeL}\left(r^{2}=0.32, p=0.011\right)$ were positively correlated with the mean change in HR over the $15 \mathrm{~min}$ following i.v. infusions (see Table 1 and Supplementary Table S2).

Although c-Fos responses to lactate $+\mathrm{L}-\mathrm{AG}$ tended to occur throughout the rostro-caudal extent of the amygdala (with the exception being the BLA, where responses were clearly restricted to the caudal part of the nucleus), these responses did not reach statistical significance at all levels nor did they correlate with changes in SI behavior or cardio-respiratory responses.

Dorsomedial medulla (DMM) systems ( $n T S, D M V)$ : As observed in the $1 \mathrm{DMH}$, rats with lactate infusions had increased c-Fos responses within the DMM (ie nTS, dorsal motor nucleus of the vagus (DMV)) of 'panic-prone' (L-AGtreated) rats but not D-AG-treated rats (Table 1 and Supplementary Figure S2c-d). Furthermore, when data from all rats in the study were considered, c-Fos responses in the nTS at $-13.68 \mathrm{~mm}$ bregma were positively correlated with the mean change in cardiovascular responses following i.v. infusions (HR $\left(r^{2}=022, p=0.040\right)$; MAP $\left(r^{2}=0.23\right.$, $p=0.044$ ); Table 1). Responses in the DMV were positively correlated with $\mathrm{RR}$ responses following i.v. infusions $\left(r^{2}=0.30, p=0.033\right)$. a
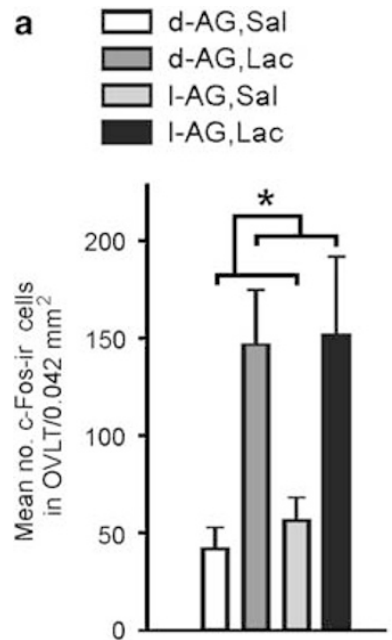

b

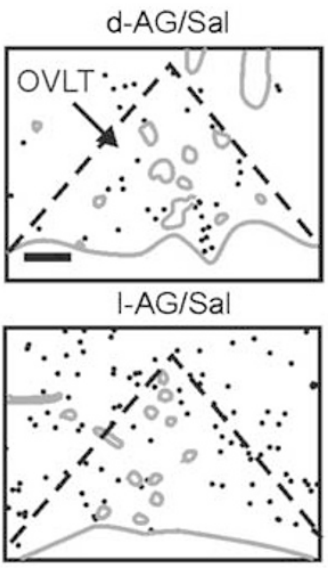

$\operatorname{OVLT}(0.00 \mathrm{~mm}$ bregma $)$

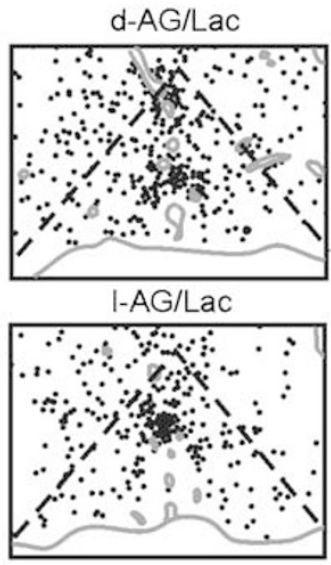

Figure 2 Graphs and illustrations of c-Fos-ir cells in the organum vasculosum lamina terminalis (OVLT) from experiment I. (a) Graph illustrates mean $( \pm$ SEM) numbers of c-Fos-ir cells in the OVLT following i.v. infusions of saline (Sal) or lactate (Lac) in rats previously treated with D- or L-AG infusions into the DMH/PeF region (*p < 0.05). (b) Illustrations of c-Fos-ir cells (each black dot = I c-Fos-ir cell) in the OVLT of a rat in each treatment group. Gray solid lines indicate the outline of the brain section and blood vessels. Black dashed lines indicate the OVLT. Scale bar: I $36 \mu \mathrm{m}$. 
Table I Mean numbers of single c-Fos-ir cells in anatomically defined subdivisions of the central nervous system following either i.v. infusions of saline (Sal) or sodium lactate (Lac) in rats with prior infusions of D- or L-AG into the DMH region from experiment I (see Supplementary Table S2 for rostral amygdala data)

\begin{tabular}{|c|c|c|c|c|c|}
\hline Single c-Fos-ir cells & d-AG/Sal & d-AG/Lac & I-AG/Sal & I-AG/Lac & \\
\hline \multicolumn{6}{|l|}{ CVOs } \\
\hline OVLT ( $0.00 \mathrm{~mm}$ bregma) & $42 \pm 11$ & $147 \pm 28$ & $56 \pm 12$ & $152 \pm 40$ & $\square$ \\
\hline AP $(-13.80 \mathrm{~mm}$ bregma $)$ & $4 \pm 1$ & $29 \pm 12$ & $3 \pm 1$ & $46 \pm 19$ & $\square$ \\
\hline \multicolumn{6}{|l|}{$\mathrm{DMH}(-3.14 \mathrm{~mm}$ bregma $)$} \\
\hline IDMH ipsi & $48 \pm 17$ & $50 \pm 16$ & $57 \pm 8$ & $105 \pm 9 c$ & $\# \ddagger$ \\
\hline $\mathrm{mDMH}$ contra & $40 \pm 12$ & $63 \pm 17$ & $74 \pm 5$ & $99 \pm 20$ & $\square$ \\
\hline DA contra & $45 \pm 13$ & $44 \pm 12$ & $67 \pm 10$ & $99 \pm 13 c$ & $\dagger \boldsymbol{\square}$ \\
\hline IDMH contra & $63 \pm 15$ & $59 \pm 13$ & $96 \pm 11$ & $97 \pm 17$ & 口 \\
\hline \multicolumn{6}{|l|}{ PVN (-1.80 mm bregma) } \\
\hline SON (-I.80 mm bregma) & $88 \pm 35$ & $146 \pm 35$ & $91 \pm 26$ & $312 \pm 71 b$ & $\dagger \ddagger \boldsymbol{\square}$ \\
\hline \multicolumn{6}{|l|}{ Amygdala ( $-2.56 \mathrm{~mm}$ bregma) } \\
\hline $\mathrm{CeC}$ & $70 \pm 31$ & $89 \pm 18$ & $66 \pm 19$ & $160 \pm 28$ & $\dagger \mathbf{\square}$ \\
\hline CeL & $28 \pm 6$ & $62 \pm 18$ & $34 \pm 13$ & $116 \pm 17 b$ & $\dagger \boldsymbol{\square}$ \\
\hline $\mathrm{CeM}$ & $42 \pm 14$ & $37 \pm 8$ & $36 \pm 6$ & $62 \pm 11$ & $\square$ \\
\hline BLA & $70 \pm 13$ & $65 \pm 16$ & $93 \pm 11$ & $109 \pm 11$ & $*$ \\
\hline BLP & $19 \pm 5$ & $17 \pm 3$ & $15 \pm 1$ & $34 \pm 11$ & $\square$ \\
\hline LA & $68 \pm 19$ & $81 \pm 24$ & $83 \pm 12$ & $119 \pm 21$ & $\square$ \\
\hline \multicolumn{6}{|l|}{ BNST (+0.20 mm bregma) } \\
\hline \multicolumn{6}{|l|}{ BNST (-0.26 mm bregma) } \\
\hline LSV & $41 \pm 12$ & $68 \pm 26$ & $68 \pm 15$ & $139 \pm 27$ & 口 \\
\hline PBN (-9.30 mm bregma) & $109 \pm 28$ & $120 \pm 29$ & $87 \pm 10$ & $419 \pm 80 b, c$ & \#ם \\
\hline \multicolumn{6}{|l|}{$R P a$} \\
\hline$(-11.30 \mathrm{~mm}$ bregma $)$ & $26 \pm 5$ & $64 \pm 20$ & $27 \pm 6$ & $46 \pm 8$ & $\square$ \\
\hline (-11.60 mm bregma) & $14 \pm 4$ & $43 \pm 11 \mathrm{a}$ & $16 \pm 4$ & $15 \pm 5$ & $\square$ \\
\hline \multicolumn{6}{|l|}{ nTS } \\
\hline (-13.30 mm bregma) & $23 \pm 7$ & $77 \pm 16$ & $32 \pm 11$ & $153 \pm 54$ & $\square$ \\
\hline (-13.68 mm bregma) & $33 \pm 7$ & $74 \pm 14$ & $18 \pm 4$ & $144 \pm 29 b, c$ & $\dagger \ddagger \boldsymbol{\square}$ \\
\hline$(-14.20 \mathrm{~mm}$ bregma $)$ & $11 \pm 2$ & $33 \pm 13$ & $14 \pm 7$ & $49 \pm 20$ & $\square$ \\
\hline DMV (- I3.68 mm bregma) & $6 \pm 2$ & $17 \pm 6$ & $4 \pm 2$ & $29 \pm 14$ & $\# \square$ \\
\hline
\end{tabular}

Abbreviations: AP, area postrema; AG, allylglycine; BLA, basolateral amygdala; BSTL, the lateral BNST; BSTM, the medial BNST; DMH, dorsomedial hypothalamus; DMV, dorsal motor nucleus of the vagus; LSV, ventral part of the lateral septum; nTS, nucleus of the solitary tract; OVLT, organum vasculosum of the lamina terminalis; PBN, parabrachial nucleus; PVN, paraventricular hypothalamic nucleus; RPa, raphe pallidus; SFO, subfornical organ; SON, supraoptic nucleus. $\mathrm{a}=$ vs d-AG/Sal; $\mathrm{b}=$ vs I-AG/Sal; c =vs d-AG/Lac; $\square$ non-responsive; $\square$ Lac only; $\mathbf{\square}$ I-AG only; $\mathbf{\square}$ Lac+I-AG and/or correlates with panic-like responses; $\dagger=$ correlates with HR following i.v. infusions; $\ddagger=$ correlates with MAP following i.v. infusions; \# = correlates with RR following i.v. infusions; * $=$ inversely correlates with change in SI from pre I-AG to post I-AG treatment.

Column I indicates brain regions; numbers in parentheses indicate anatomical levels relative to bregma. Columns $2-5$ represent a) the number of c-Fos-ir cells. The titles above columns indicate treatment groups, D-AG/Sal, D-AG/Lac, L-AG/Sal, or L-AG/Lac, respectively. Values below columns $2-5$ are the mean \pm SEM. Letters, symbols, and boxes adjacent to column 5 are defined in figure legend in table. 
Table 2 Mean numbers of c-Fos-ir cells in anatomically and/or chemically defined subdivisions of the central nervous system following either i.v. infusions of saline (Sal) or sodium lactate (Lac) in rats with prior infusions of D- or L-allylglycine (D- or L-AG) into the DMH region from experiment I

\begin{tabular}{|c|c|c|c|c|}
\hline & d-AG/Sal & $d-A G / L a c$ & I-AG/Sal & I-AG/Lac \\
\hline \multicolumn{5}{|l|}{ a. c-Fos/TH-ir neurons } \\
\hline \multicolumn{5}{|l|}{ Pons } \\
\hline A6 (- $10.04 \mathrm{~mm}$ bregma) no. c-Fos/TH & $29 \pm 9$ & $85 \pm 18$ & $32 \pm 12$ & $74 \pm 25 \square$ \\
\hline \multicolumn{5}{|l|}{ Ventrolateral medulla } \\
\hline $\mathrm{Cl}$ (-12.72 mm bregma) no. c-Fos/TH & $6 \pm 2$ & $17 \pm 2 \mathrm{a}$ & $6 \pm 1$ & $23 \pm 4 b \square$ \\
\hline $\mathrm{AICl}$ (-13.68 mm bregma) no. c-Fos/TH & $15 \pm 4$ & $20 \pm 3$ & $20 \pm 5$ & $25 \pm 3 \square$ \\
\hline \multicolumn{5}{|l|}{ Dorsomedial medulla } \\
\hline DMV (I $3.68 \mathrm{~mm}$ bregma) no. c-Fos/ChAT & $\mid \pm 1$ & $\mid \pm 1$ & $0 \pm 0$ & $3 \pm 2 \square$ \\
\hline Amb (I I.80 mm bregma) no. c-Fos/ChAT & $0 \pm 0$ & $0 \pm 0$ & $0 \pm 0$ & $0 \pm 0 \square$ \\
\hline \multicolumn{5}{|l|}{ c. c-Fos/NeuN-ir neurons } \\
\hline PBN ( $9.30 \mathrm{~mm}$ bregma) no. c-Fos/NeuN & $16 \pm 4$ & $16 \pm 4$ & $5 \pm 1$ & $80 \pm 22 \square$ \\
\hline
\end{tabular}

Abbreviations: AG, allylglycine; ChAT, choline acetyltransferase; NeuN, neuronal marker $\mathrm{n}$; TH, tyrosine-hydroxylase.

$\mathrm{a}=\mathrm{vs} \mathrm{d}-\mathrm{AG} / \mathrm{Sal}$; $\mathrm{b}=\mathrm{vs}$ I-AG/Sal; $\square$ non-responsive; $\square$ Lac only; $\mathbf{\square}$ Lac+l-AG and/or correlates with panic-like responses.

For table (a), (b), and (c) column I indicates brain regions; numbers in parentheses indicate anatomical levels relative to bregma. Columns 2-5 represent (a) the number of c-Fos/TH-ir neurons; (b) the number of c-Fos/ChATir neurons; and (c) the number of c-Fos/NeuN-ir neurons. No significant differences in the total number of TH, ChAT, or NeuN-ir neurons were observed between groups in any subregion analyzed (see the RESULTS section for details). The titles above columns $2-5$ for (a), (b), and (c) indicate treatment groups, D-AG/Sal, D-AG/Lac, L-AG/Sal, or L-AG/Lac, respectively. Values below columns 2-5 are the mean \pm SEM. Letters, symbols, and boxes adjacent to column 5 are defined in figure legend in table.

In addition to overall c-Fos analysis, c-Fos expression in the acetylcholinergic parasympathetic preganglionic neurons (c-Fos + ChAT) of the DMV and the Amb were investigated. Consistent with the previous immunohistochemical analysis, lactate infusion, compared to vehicle infusion, increased numbers of c-Fos-ir cells in the DMV of L-AG-, but not D-AG-treated rats (lactate effect; $\mathrm{F}_{(1,20)}=11.05, p=0.003$; Table 2b). However, these increases in c-Fos were not occurring in ChAT-ir neurons in either the DMV or Amb (Table $2 b$ and Figure $3 a-b$, respectively). No differences in the total numbers of ChATir cells within the DMV or Amb were observed among treatment groups.

To determine if the increases in c-Fos expression in these regions were in local GABAergic inhibitory neurons, we performed double immunostaining for GAD67 and c-Fos. Dense synaptic bouton-like staining, as evidenced by small GAD67-ir granules, made visualizing and accurately counting c-Fos/GAD67 double-ir cells difficult and thus a quantitative analysis was not performed. However, there were examples of c-Fos/GAD67-ir cells noted in the nTS of $\mathrm{L}-\mathrm{AG}+$ lactate-treated rats (Figure 3c).
Paraventricular hypothalamic nucleus (PVN) and supraoptic nucleus (SON): Within the PVN, L-AG+ lactate-treated rats had increased c-Fos responses in the dorsal cap and ventral PVN (PaDC and $\mathrm{PaV}$, respectively; Table 1 and Supplementary Figure S3a,c) compared to other groups. However, c-Fos responses in the PaDC did not correlate with behavioral or cardiorespiratory responses. Lactate infusions also increased c-Fos responses in the lateral magnocellular and medial parvicellular PVN (PaLM and PaMP, respectively) in both L-AG- and D-AG-treated rats. Lactate increased c-Fos responses in the SON, but only in L-AG-treated rats (Table 1). Furthermore, c-Fos responses in the SON were positively correlated with HR $\left(r^{2}=0.42, p=0.003\right)$ and MAP $\left(r^{2}=0.37, p=0.007\right)$ following i.v. infusions.

Parabrachial nucleus (PBN): Sodium lactate increased c-Fos responses in the PBN (Table 1, Supplementary Figure $\mathrm{S} 3 \mathrm{~b}, \mathrm{~d})$ but only in L-AG-treated rats. In addition, the number of c-Fos-ir cells in the PBN was positively correlated with $\mathrm{RR}\left(r^{2}=0.26, p=0.027\right)$, but not $\mathrm{HR}$, MAP, or SI duration responses following i.v. infusions (see Table 1). 


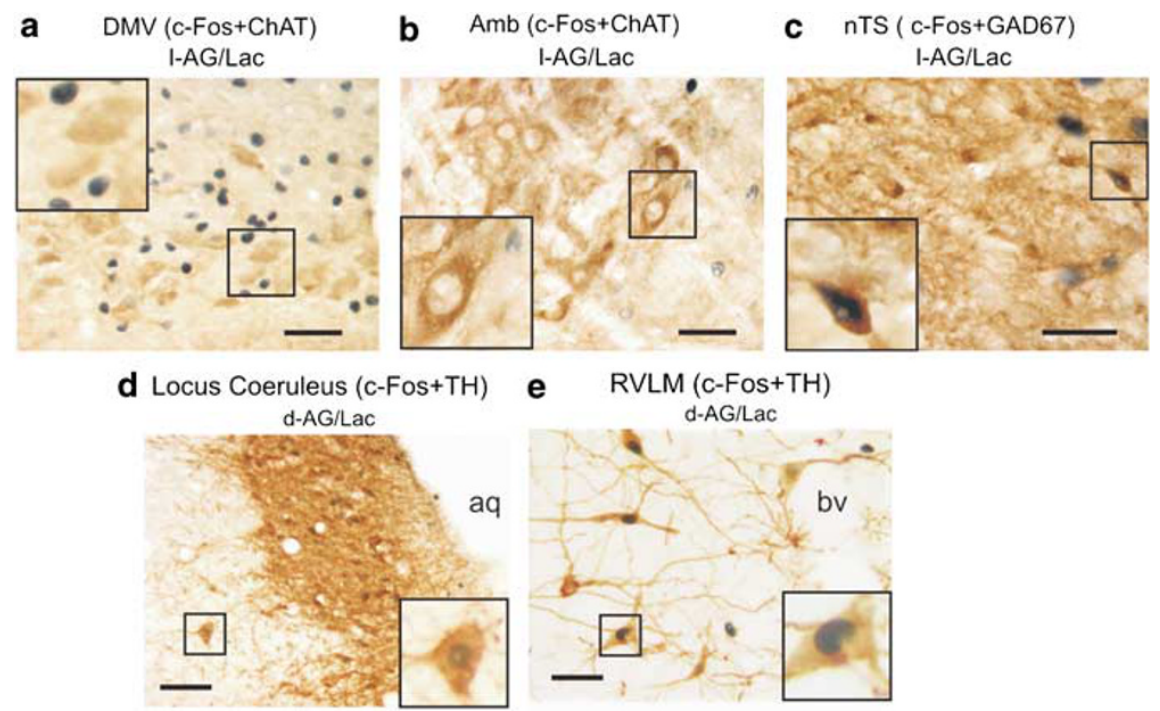

Figure 3 Photomicrographs of cellular responses (ie c-Fos induction; blue/black immunostaining of nuclei) in neurochemically identified cell groups studied in experiment I represented brown cytoplasmic immunostaining. Photographs of c-Fos and choline acetyltransferase (ChAT) immunostaining in the (a) dorsal motor nucleus of the vagus (DMV) and (b) ambiguus nucleus (Amb) in lactate (Lac) + L-AG-treated rats. Photographs of (c) c-Fos and glutamic acid decarboxylase (GAD67) immunostaining in the nucleus of the solitary tract (nTS) of a lactate + L-AG-treated rat. Photomicrographs illustrating c-Fos and tyrosine hydroxylase $(\mathrm{TH})$ immunostaining of (d) pontine A6 noradrenergic cell group in the locus coeruleus (LC, $-10.04 \mathrm{~mm}$ bregma) and (e) rostroventrolateral medulla (RVLM) CI adrenergic cell group (-11.96 mm bregma) in saline- (Sal, left) and Lac (right) infused rats with prior infusions of Dallylglycine (D-AG) in the DMH/PeF. Insets at the bottom of low magnification photographs contain higher magnification photographs. Scale bar: $50 \mu$ m; lower left insert; (a, b) $25 \mu \mathrm{m}$, (c) $22 \mu \mathrm{m}$, (d, e) $20 \mu \mathrm{m}$.

An estimation of c-Fos induction in neurons in a brain region that showed a robust $\mathrm{L}-\mathrm{AG}+$ lactate effect (ie the $\mathrm{PBN}$ ) revealed that $\mathrm{L}-\mathrm{AG}+$ lactate induction of $\mathrm{c}$-Fos in the ventrolateral PBN was exclusively occurring in neurons (see Supplementary Table S1d and Table 2c). There was no evidence for increased c-Fos expression in non-neuronal cells.

Other CNS regions that were non-responsive or where responses did not correlate with panic-like responses: Surprisingly, no subdivision of the midbrain periaqueductal gray (PAG), or ventrolateral medulla (ie Amb, PreBo, LRt, LPGi, caudal ventrolateral medulla (CVLM), and rostroventrolateral medulla (RVLM) (with the exception of $\mathrm{C} 1$ adrenergic neurons)) responded to any independent variable (see Supplementary Table S3). An increased c-Fos response was observed in the raphe pallidus ( $\mathrm{RPa}$ ) in response to lactate, but only in D-AG-treated control rats (see Table 1). As predicted control sites such as the hypoglossal nucleus (12) expressed low levels of c-Fos in all treatment groups (see Table 1).

Pontine and medullary noradrenergic and adrenergic systems: Lactate-treated rats had altered c-Fos responses in specific noradrenergic and adrenergic ( $\mathrm{TH}-\mathrm{ir})$ cell groups involved in central autonomic control. Overall, the numbers of TH-ir neurons within neuronal cell groups were largely unaffected by either L-AG or lactate infusion (Table 2a and Supplementary Table S4).

Lactate infusions increased the number of c-Fos/TH-ir neurons in the A6 cell group (LC) at $-10.04 \mathrm{~mm}$ bregma in D-AG-, but not L-AG, -treated rats (Table 2a and
Supplementary Table S4, Figure 3d). Neither lactate nor $\mathrm{L}-\mathrm{AG}$ infusions altered the number of c-Fos/TH-ir neurons in any other pontine noradrenergic cell group (see Supplementary Figure S1).

Lactate infusions increased the number of c-Fos/TH-ir neurons throughout the rostrocaudal extent of both the C1 (located in the RVLM; Table 2a, Figure $3 \mathrm{e}$ and Supplementary Table S4) and C2 (located in the DMM; Table 2a and Supplementary Table S4) cell groups in both L-AG- and D-AG-treated rats. Neither L-AG nor lactate altered the number of c-Fos/TH-ir neurons in the $\mathrm{C} 3, \mathrm{~A} 1 / \mathrm{C} 1$, or $\mathrm{A} 1$ cell groups (see Supplementary Figure $\mathrm{S} 1$ ).

Intra- and inter-rater analyses of c-Fos-ir cell counts: Intra- and inter-rater analyses of c-Fos-ir cell counts for the OVLT and the CeA revealed that correlations between cell counts were highly significant. The intra-rater analyses of c-Fos counts revealed an $r^{2}=0.94, p<0.001$ for the OVLT; $r^{2}=0.97$ and $p<0.001$ for the CeA. The inter-rater analyses of c-Fos counts revealed an $r^{2}=0.88, p<0.001$ for the OVLT and an $r^{2}=0.98, p<0.001$ for the CeA (data not shown).

\section{Experiment 2: Restoring GABAergic Tone in the DMH/ PeF Blocks L-Ag + Lactate-Induced Panic Responses}

To determine if restoring GABA signaling in the $\mathrm{DMH} / \mathrm{PeF}$ of L-AG-treated rats was sufficient to block the lactateinduced panic-like effects, injections of muscimol, a $\mathrm{GABA}_{\mathrm{A}}$ receptor agonist, into the $\mathrm{DMH} / \mathrm{PeF}$ region $30 \mathrm{~min}$ before the onset of lactate infusion blocked $\mathrm{L}-\mathrm{AG}+$ lactate-induced increases in $\mathrm{HR} \quad\left(\mathrm{F}_{(3,20)}=50.2, p<0.001\right)$ and $\mathrm{RR}$ 
a

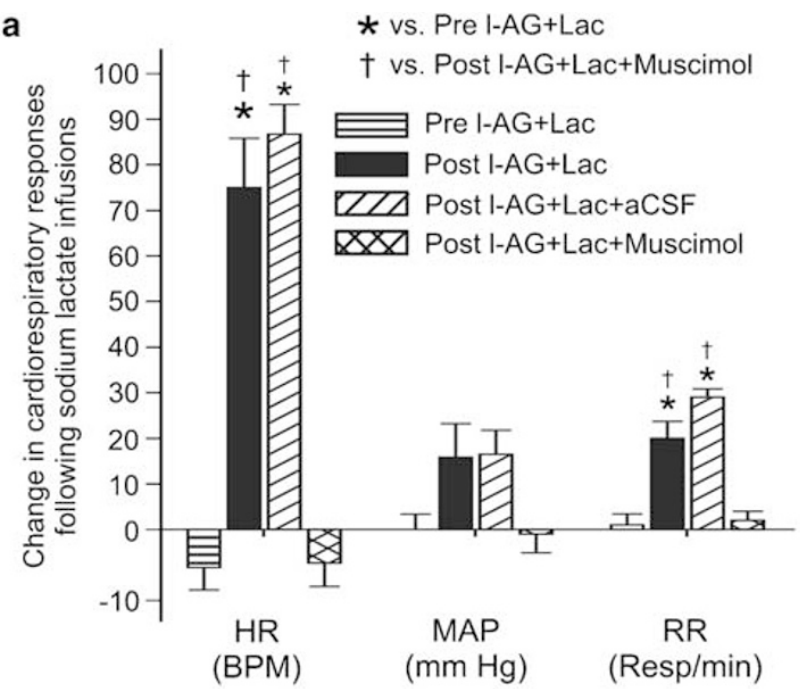

-2.80 to -3.30 $\mathrm{mm}$ bregma
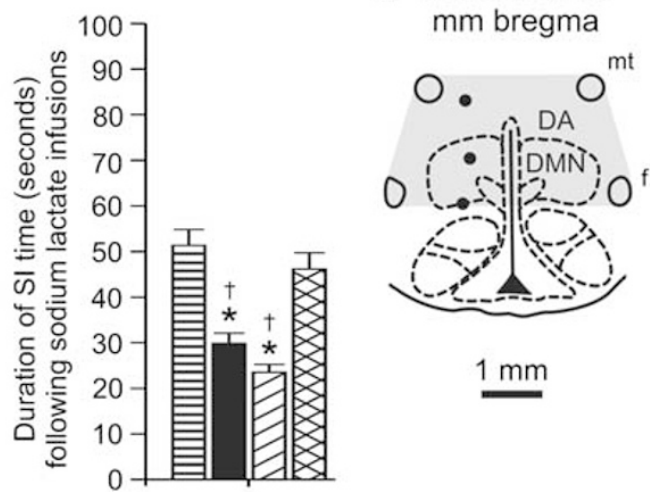

C
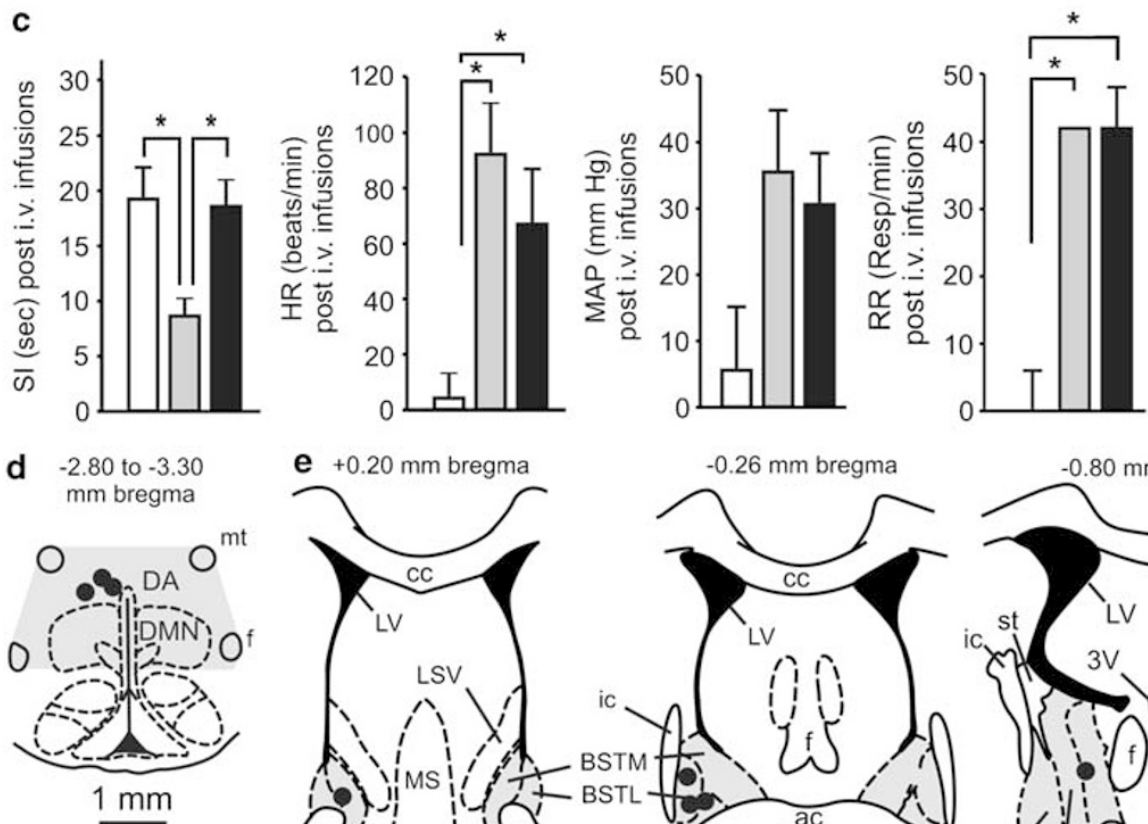

$\square$ Vehicle in BNST
(Pre I-AG+Lac)
$\square$ Vehicle in BNST Vehicle in $B$
$(1-A G+L a c)$

Muscimol in BNST (I-AG+Lac)
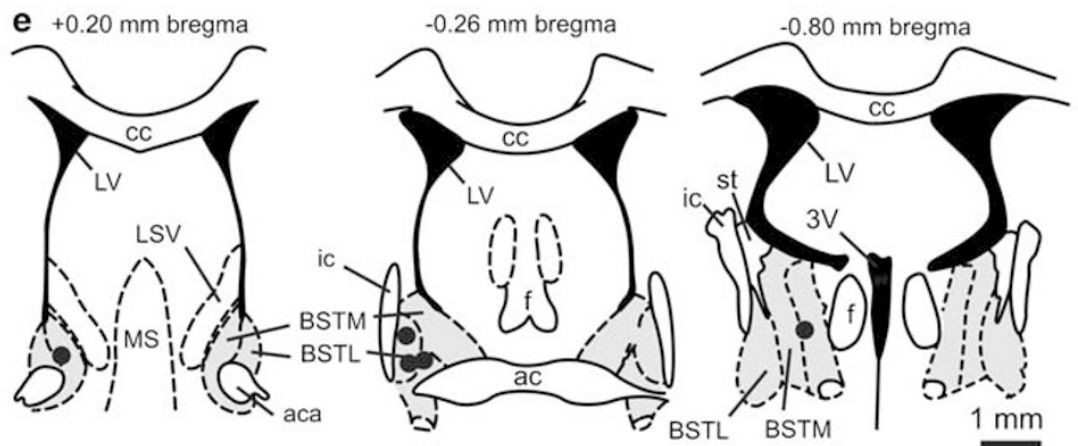

Figure 4 Effects of restoring GABAergic tone in the DMH (experiment 2) or bed nucleus of the stria terminalis (BNST, experiment 3) on L-AG + lactate (Lac)-induced behavioral and/or cardiorespiratory responses. (a) Graph illustrates the effects of injecting muscimol or artificial cerebrospinal fluid (aCSF) into the DMH region of L-AG + Lac-treated rats. Bars represent the mean \pm SEM (* (vs Pre L-AG + Lac) $p<0.00 I,{ }^{\dagger}$ (vs Post-L-AG + Lac + Muscimol) $p<0.0 I$ )). (b) Illustration of cannula placements in the DMH region for experiment 2. Black circles represent cannula placements verified histologically $(n=3)$ and gray shading indicates cannula placements from tissue that was micropunched for future neurochemical analyses $(n=3)$. (c) First graph, SI duration prior to and after L-AG infusions with aCSF or muscimol injections targeting the BNST followed by i.v. sodium lactate infusions. The following graphs illustrate the changes in the MAP, HR and RR in response to lactate prior to and after L-AG infusions in the DMH and with aCSF or muscimol injections targeting the BNST in experiment 3; $(* p<0.05)$. (d) Illustration of cannula placements in the DMH region for experiment 3. Black circle represents one histological placement $(n=3)$ and gray shading represents distribution of cannula placements from tissue micropunched for neurochemical analysis $(n=2)$. (e) Illustration of the distribution injection sites in the BNST in experiment 3. Gray shading represents the BNST and black circles indicate aCSF or muscimol injection sites $(n=5)$ verified with histology. See Materials and Methods section for full list of abbreviations.

$\left(\mathrm{F}_{(3,20)}=28.4, \quad p<0.001\right)$ and blocked $\mathrm{L}-\mathrm{AG}+$ lactate-induced decreases in SI duration (Figure 4a; $\mathrm{F}_{(3,20)}=21.2$, $p<0.001)$. The $\mathrm{L}-\mathrm{AG}+$ lactate treatment altered the MAP $\left(\mathrm{F}_{(3,20)}=3.4, p=0.039\right)$, but Tukey's did not detect a significant change among groups. Pharmacological and histological analyses, as detailed in methods, confirmed successful cannulae implantation in the DMH/PeF region (Figure $4 \mathrm{~b}$ ).

\section{Experiment 3: The BNST Regulates Anxiety-like Responses in Panic Model}

To determine if excitation of the BNST is involved with the anxiety-like responses but not cardiorespiratory responses to sodium lactate challenge in L-AG-treated rats, muscimol or vehicle injections were administered to the BNST $30 \mathrm{~min}$ prior to a lactate challenge in L-AG-treated rats. As reported 
in experiments 1-3, L-AG-treated rats had reduced SI duration $\left(\mathrm{F}_{(2,11)}=6.0, p=0.017, n=5\right)$ and increased $\operatorname{HR}\left(\mathrm{F}_{(2,11)}=9.3, p=0.004, n=5\right)$ and $\mathrm{RR}\left(\mathrm{F}_{(2,3)}=24.5\right.$, $p=0.014, n=2)$ but not $\operatorname{MAP}\left(\mathrm{F}_{(2,10)}=3.3, p=0.078, n=4\right)$ in response to lactate, compared to pre-L-AG baseline measurements (Figure 4c). Post hoc tests revealed that unilateral injections of $250 \mathrm{pmol}$ muscimol ( $100 \mathrm{nl})$, but not vehicle, into the BNST blocked L-AG-induced decreases in SI duration in the presence of lactate but had no effect on lactate-induced increases in HR or RR in L-AG-treated rats (Figure 4c). Histological examination confirmed successful implantation of cannulae in the $\mathrm{DMH} / \mathrm{PeF}$ region (Figure $4 \mathrm{~d}$ ) and in the BNST region (Figure $4 \mathrm{e}$ ).

\section{Experiment 4: Intravenous Atenolol Infusions Block Tachycardia Responses}

In order to verify that a peripheral sympathetic response occured in lactate $+\mathrm{L}-\mathrm{AG}$-treated rats, systemic application of the selective $\beta 1$-adrenergic blocker, atenolol was administered i.p. prior to the lactate challenge in 'panicprone' (L-AG-treated) rats. As reported above, challenging L-AG-treated rats with lactate increased cardiovascular responses (MAP, time effect, $\mathrm{F}_{(19,100)}=3.3, p<0.001$ ( 5 min baseline +15 min post-infusion); HR, time effect, $\mathrm{F}_{(9,50)}=3.2, p<0.005$ (only when comparing 5 min baseline +5 min post-infusion)) (see Supplementary Figure S4a,b). Another group of $\mathrm{L}-\mathrm{AG}+$ lactate-treated rats that also received i.v. atenolol had the expected hypertension (time effect, $\mathrm{F}_{(19,80)}=1.8, p<0.05$ (5 min baseline +15 min postinfusion)), but failed to have a tachycardia response (time effect, $\mathrm{F}_{(11,48)}=0.2, p=0.997$ ( $5 \mathrm{~min}$ baseline $+5 \mathrm{~min}$ postinfusion)). Treating $\mathrm{L}-\mathrm{AG}+$ saline-treated rats with i.v. atenolol did not alter MAP (time effect, $\mathrm{F}_{(19,80)}=0.6$, $p=0.888$ ( $5 \mathrm{~min}$ baseline $+15 \mathrm{~min}$ post-infusion) $)$ or $\mathrm{HR}$ (MAP, time effect, $\mathrm{F}_{(11,48)}=0.4, p=0.950$ (5 min baseline + 5 min post-infusion)) responses from baseline. The mean baseline values for each treatment group did not differ (see Supplementary Figure S4a-b for baselines). Histological examination confirmed successful implantation of cannulae in the DMH/PeF region (Supplementary Figure S4c).

\section{DISCUSSION}

\section{$\mathrm{DMH} / \mathrm{PeF}$ as one Putative site Coordinating Panic-Like Responses}

The hypothalamus has long been implicated in generating a coordinated emotional arousal (see review (DiMicco et al, 2002)). More specifically, blockade of inhibitory GABA neurotransmission in the $\mathrm{DMH} / \mathrm{PeF}$ elicits increases in $\mathrm{HR}$ and RR, BP, plasma catecholamine, adrenocorticotrophic hormone, and corticosterone concentrations, intestinal and colonic motility, and a pattern of peripheral blood flow that is similar to the pattern of peripheral blood flow during the defense reaction (see review (DiMicco et al, 2002)). This pattern of autonomic responses is similar to that seen during panic attacks in humans (Liebowitz et al, 1986). In addition to the autonomic, respiratory, and endocrine responses, activation of the $\mathrm{DMH} / \mathrm{PeF}$ also elicits escape behaviors (Shekhar and DiMicco, 1987), selective enhancement of fear responses (Shekhar et al, 1987) and anxiety-like behavior as measured by the conflict (Shekhar et al, 1990), EPM (Shekhar, 1993) and SI tests (Shekhar and Katner, 1995) in rats. The known neuroanatomical connections of the $\mathrm{DMH} / \mathrm{PeF}$ are consistent with its proposed key integrative role in anxiety and panic-like responses. Thus, $\mathrm{DMH} / \mathrm{PeF}$ contains a neuronal system which is essential for behavioral and cardiorespiratory responses associated with emotional arousal. A defect in such a regulatory mechanism could result in susceptibility to panic attacks. In support of these hypotheses, we previously demonstrated in experiment 1 that only panic-prone rats infused with sodium lactate had increased cellular responses in the $\mathrm{DMH} / \mathrm{PeF}$ which also correlated with cardio-respiratory responses (Johnson and Shekhar, 2006). We have previously demonstrated that the increased cellular responses in the DMH/ $\mathrm{PeF}$ are almost exclusively occurring in NMDA, but not AMPA, receptor subunit expressing cells, with a morphology (ie size and shape) consistent with a neuronal phenotype (Johnson and Shekhar, 2006). Since most glia express AMPA receptor subunits, this suggests that the cFos induction in the $\mathrm{DMH} / \mathrm{PeF}$ is occurring in a unique set of neurons (Seifert et al, 1997). Furthermore, injecting specific NMDA receptor antagonists into the DMH of $\mathrm{L}-\mathrm{AG}-$ treated rats prior to a lactate challenge blocks all anxietylike behavioral and panic-like cardiorespiratory responses (Johnson and Shekhar, 2006). The neurotransmitter phenotype of these $\mathrm{DMH}$ cells remains uncertain, but there are some enticing candidates which may be involved. Neurons that synthesize the neuropeptide orexin express the NMDA receptor (see review (Winsky-Sommerer et al, 2004)) and are only found in the $\mathrm{DMH}$ and adjacent hypothalamus (Peyron et al, 1998). Central orexin production is critical for maintaining wakefulness and wakefulness and vigilance (see review (Sakurai, 2007)) and orexin has been demonstrated to mobilize centrally mediated sympathetic responses (Ferguson and Samson, 2003) which is a key component of panic. Overall, this suggests that chronic infusions of $\mathrm{L}-\mathrm{AG}$ into the $\mathrm{DMH} / \mathrm{PeF}$ are inducing panic-prone states by making the $\mathrm{DMH} / \mathrm{PeF}$ susceptible to mobilize panic-like responses to a subthreshold interoceptive cue (ie $0.5 \mathrm{M}$ sodium lactate). In the present article, this was verified in experiment 2 by injecting muscimol into the $\mathrm{DMH} / \mathrm{PeF}$ of L-AG-treated rats prior to lactate and blocking all aspects of L-AG + lactate-induced panic-like responses. Therefore, the present study clearly demonstrates a critical role for the $\mathrm{DMH} / \mathrm{PeF}$ region in acutely eliciting panic-like responses following lactate infusions.

\section{Pathways for Lactate-Induced Panic-Like Responses}

A critical component underlying sodium lactate's ability to induce panic-like cardiorespiratory responses in this panic model involves interactions between the CVOs (OVLT/ SFO), areas in the CNS that lack a blood-brain barrier and therefore serve as 'sensors' of changes in osmolarity, $\mathrm{pH}$ and other plasma parameters (Johnson and Gross, 1993), and the $\mathrm{DMH} / \mathrm{PeF}$ which is a major target of these CVOs (Thompson and Swanson, 1998). Based on this, neurons in the CVOs are likely to react to sodium lactate infusions (Johnson and Gross, 1993) and propagate this signal to the $\mathrm{DMH} / \mathrm{PeF}$ in both L-AG- and D-AG-treated rats. As predicted, the lactate challenge increased c-Fos responses 
in the OVLT of panic-prone and non-panic-prone rats equally. This suggests that the sodium lactate signal is being relayed to the $\mathrm{DMH} / \mathrm{PeF}$ regardless of $\mathrm{D}-\mathrm{AG}$ or $\mathrm{L}-\mathrm{AG}$ treatment. This also suggests that in D-AG-treated rats with intact GABAergic tone in the $\mathrm{DMH}$, the subthreshold dose of sodium lactate is not sufficient to activate the DMH. However, in L-AG-treated rats with compromised GABAergic tone in the $\mathrm{DMH} / \mathrm{PeF}$, this subthreshold signal is sufficient to activate the DMH where the signal is then further propagated to efferent targets implicated in anxiety and central autonomic control. The effect of L-AG in the hypothalamus may be specific to the $\mathrm{DMH} / \mathrm{PeF}$ since we previously demonstrated that infusing L-AG into the other surrounding regions (ie the paraventricular nucleus of the hypothalamus) does not induce panic-like responses following a sodium lactate challenge (Shekhar et al, 1996). The importance of the OVLT in the DMH/PeF panic model was previously demonstrated in a previous article where injections of a small amount $(100 \mathrm{nl})$ of lactate directly into the OVLT/AV3V region of panic-prone rats (L-AG treated), but not D-AG-treated controls, elicited panic-like responses (Shekhar and Keim, 1997). Furthermore, injecting tetrodotoxin (a voltage gated sodium channel blocker) into the OVLT region of panic-prone rats blocked i.v. lactateinduced panic-like responses (Shekhar and Keim, 1997). Overall, the OVLT/AV3V region appears to be an important site for 'sensing' plasma sodium lactate and relaying the signal to the $\mathrm{DMH} / \mathrm{PeF}$.

A subset of $\mathrm{DMH} / \mathrm{PeF}$ efferent pathways appears to be activated during the panic-like response induced by lactate infusions. Among the forebrain limbic structures, the BNST, LSV, and several subnuclei of the amygdala were selectively activated during panic-like responses. The c-Fos induction in the rostral BNST region correlated strongly with anxietylike responses, whereas c-Fos induction in the caudal BNST correlated with panic-like cardiovascular responses. Although other studies have demonstrated that the BNST regulates anxiety-like behavior (Sajdyk et al, 2007) and autonomic activity (Crestani et al, 2007), the BNST's role may depend on the context. In the present article, a subsequent experiment (ie experiment 3 ) demonstrated that inactivation of the rostro-caudal BNST region (see Figure 4) with acute muscimol injections selectively blocked the anxiogenic effects of the lactate $+\mathrm{L}-\mathrm{AG}$ infusions without affecting the cardiorespiratory activation, providing strong support for the hypothesis that the BNST selectively regulates anxiety behavior, but not panic-like cardiorespiratory responses in the panic model presented here (see Figure 5a for a hypothetical model).

Although fear conditioning has not been studied in this panic model, the strong CeA responses in rats displaying increased cardiorespiratory responses could potentially be involved in laying down conditioned fear memory (see review (Davis and Shi, 1999)) that may be analogous to secondary phobia following initial panic attacks in panic disorder patients (Starcevic et al, 1993, a, b; ). Therefore, we plan in future studies to test for fear conditioning in this model (eg using conditioned place-avoidance (Thielen and Shekhar, 2002)) and, in the case that conditioning is occurring, explore the role of the CeA in this conditioning. A final comment is that removing GABAergic tone in the DMH was sufficient to increase anxiety-like behavior and a Hypothetical model for DMH mobilization of anxiety-like behavior

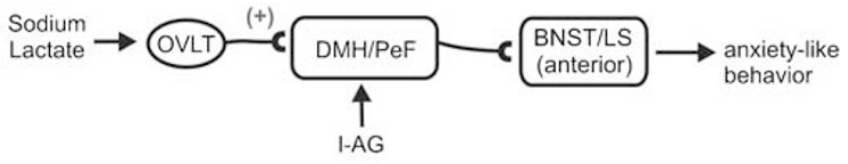

b Hypothetical model for DMH mediated sympathetic mobilization

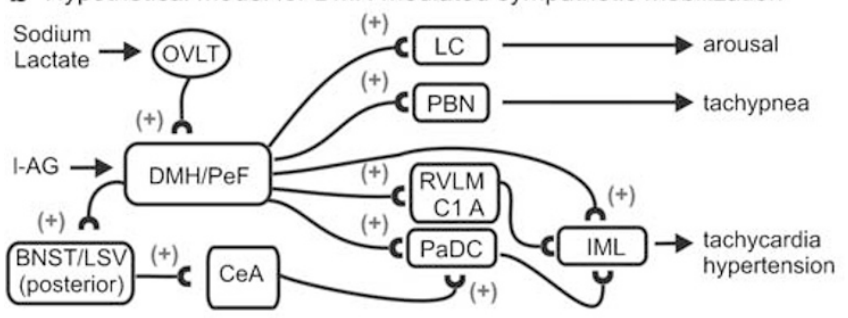

C Hypothetical model of DMH mediated desensitization of baroreflex

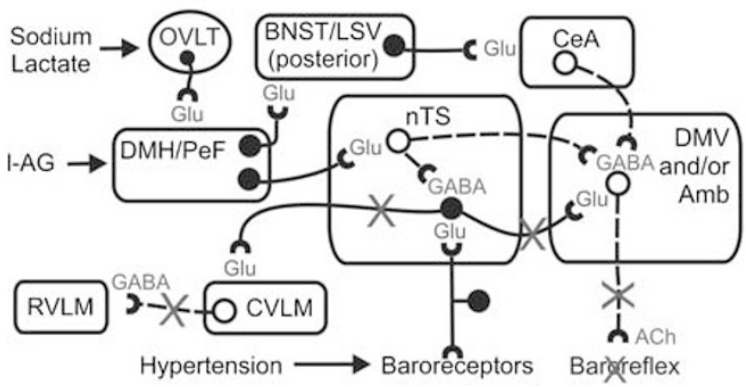

Figure 5 Hypothetical models of sodium lactate induced increases in panic-like behavioral and cardiorespiratory responses in panic-prone (ie L-AG treated) rats. (a) Hypothetical model illustrating the neural pathways of lactate + L-AG-induced anxiety where plus signs indicate an excitatory pathway. (b) Illustration of a hypothetical model of sodium lactate + L-AG mobilization of sympathetic nervous system activity. (c) Hypothetical model illustrating sodium lactate + L-AG-induced desensitization of the vagally mediated baroreflex, where solid lines with solid circles indicate excitatory neurons and dashed lines with open circles indicate inhibitory neurons and an ' $X$ ' over a line indicates paths that are inhibited by the dorsomedial hypothalamus/perifornical region (DMH/PeF). Abbreviations: bv, blood vessel; GABA, GABAergic path; Glu, glutamatergic path; IML, intermediolateral cell column; See the Materials and Methods section for additional abbreviations.

the threshold of anxiety remained high following the lactate challenge. Future studies may try and determine if L-AG + lactate elicit other stress-related behaviors that correlate with panic-like cardiorespiratory responses (eg fear associated behavior).

The tachypnea noted after lactate infusions most likely is a result of activating one of several respiratory rhythm generators in the brainstem. In the present study, the PBN was the only major respiratory center that clearly responded during panic-like responses. There is ample anatomical evidence that $\mathrm{DMH} / \mathrm{PeF}$ neurons project to $\mathrm{PBN}$ and therefore could stimulate respiration (Chamberlin and Saper, 1994; Chen et al, 2004).

The finding that systemic injections of atenolol (a $\beta 1$ adrenergic receptor antagonist) prevented $\mathrm{L}-\mathrm{AG}+$ lactateinduced tachycardia supports the hypothesis that peripheral sympathetic mechanisms contribute to tachycardia and hypertensive responses during lactate-induced panic. Further analyses of c-Fos responses revealed that discrete brain regions involved in sympathetic and parasympathetic 
regulation were positively correlated with cardiovascular responses (see Table 1). These regions included the dorsal cap of the PVN (ie PaDC), SON, PBN, CeA, caudal BNST, and the rostral nTS. Some of the other autonomic regulatory sites responded to lactate infusion (ie $\mathrm{RPa}$, LC noradrenergic, and $\mathrm{C} 1$ and $\mathrm{C} 2$ adrenergic neurons), while yet other brainstem regions associated with sympathetic mobilization failed to respond to lactate or L-AG (eg subdivisions of the PAG, etc). In light of the data presented here, there are several ways the DMH could potentially induce cardioexcitatory responses following lactate infusions in L-AG-treated rats (see Figure $5 \mathrm{~b}-\mathrm{c}$ for a hypothetical model for DMHmediated cardioexcitatory responses). The $\mathrm{DMH} / \mathrm{PeF}$ could drive hypertensive responses at efferent targets, such as the PaDC, SON, RVLM, or sympathetic regions of the spinal cord. The DMH/PeF directly innervates the PaDC, SON (Thompson and Swanson, 1998), and also sympathetic regions of the spinal cord (Hosoya et al, 1987). Neurons in the PaDC also directly innervate sympathetic regions of the spinal cord (Saper et al, 1976). Yet another explanation is that $\mathrm{C} 1$ adrenergic neurons were firing at a higher rate in lactate $+\mathrm{L}-\mathrm{AG}$-treated rats, relative to lactate $+\mathrm{D}-\mathrm{AG}$-treated rats, even though lactate induced a similar level of c-Fos expression in $\mathrm{C} 1$ adrenergic neurons in the RVLM similarly in both D-AG and L-AG-treated rats challenged with lactate. Consistent with a role for the RVLM/C1 adrenergic cell groups in DMH/PeF-mediated cardiovascular responses, pressor responses, elicited from disinhibition of the $\mathrm{DMH}$, can be severely attenuated by microinjecting muscimol into the RVLM (Fontes et al, 2001). Tachycardia responses following L-AG + lactate infusions are most likely being mobilized by a combination of direct and indirect projections from the $\mathrm{DMH} / \mathrm{PeF}$ to sympathetic preganglionic neurons.

In order for the sympathetic limb of the autonomic nervous system to simultaneously induce hypertension and tachycardia, the baroreflex mechanism which stimulates vagal parasympathetic output and inhibits the sympathetic output needs to be desensitized (see review (McDowall et al, 2006)). Within the DMM (a region that is critical for the baroreflex (Catelli and Sved, 1988) and contains parasympathetic motor neurons), infusions of lactate increased c-Fos-ir cells in the nTS but only in L-AG-treated rats. However, increased c-Fos responses in the DMM were not occurring in parasympathetic preganglionic acetylcholinergic neurons (DMV and Amb), or other regions involved in the baroreflex-mediated sympathetic inhibition (eg CVLM) (Chan and Sawchenko, 1998). The DMH directly innervates the nTS (Fontes et al, 2001), and electrical or chemical (see review (McDowall et al, 2006)) stimulation of the $\mathrm{DMH}$ decreases the sensitivity of the baroreflex through a GABAergic mechanism in the nTS/DMV (Jordan et al, 1988). This circuit represents an adaptive means of inhibiting the baroreflex during 'fight or flight' responses and exercise (Potts et al, 2003). The nTS contains numerous GABAergic neurons (Fong et al, 2005) which could be dampening parasympathetic activity by inhibiting acetylcholinergic vagal preganglionic neurons. This notion is supported by recent evidence that stimulation of nTS neurons in vitro inhibits DMV neurons (Davis et al, 2003; Sevoz-Couche et al, 2003). Therefore, the increased c-Fos-ir cells in the nTS could be occurring in local GABAergic neurons. We demonstrated this with double immunohistochemistry where c-Fos/GAD67-ir neurons were noted in $\mathrm{L}-\mathrm{AG}+$ lactate-treated rats, even though intense GAD67-ir fibers in the nTS and DMV prevented quantification of double immunostaining.

The finding that panic-related conditions may be a result of parasympathetic and sympathetic imbalance, and baroreflex disruption is not surprising (see review (Friedman and Thayer, 1998)). Panic disorder patients have higher baseline HR than controls (Gorman et al, 1983; Nesse et al, 1984) and electrocardiograms in panic disorder patients reveal sympathetic dominance and parasympathetic withdrawal (Hamada et al, 1998; McCraty et al, 2001). Panic disorder patients also show disrupted baroreceptor function that persists even when they are in remission (Shioiri et al, 2005). Also, supporting disruption of GABA function in panic disorder are data showing that genetic polymorphisms in the GAD genes are associated with vulnerability to panic disorder (Hettema et al, 2005). Moreover, GABA enhancers such as benzodiazepine agonists attenuate sodium lactate-induced panic-related responses in panic patients (Pohl et al, 1994), and panic patients demonstrate deficits in central GABA concentrations (Goddard et al, 2001).

Technical considerations. It is not certain whether the present anxiety-like responses and cardioexcitatory physiological responses of rats are equivalent to anxiety and panic attacks in humans, respectively. Furthermore, one must use caution when interpreting neural circuitry data based on immediate-early gene induction (Morgan and Curran, 1989). For example, it is possible that supraspinal efferent pathways from the DMH (eg PAG) were involved in sympathoexcitatory responses even though no differences in the numbers of c-Fos-ir cells were observed. The phenotype of the cells (ie neuronal or glial-like) that expressed c-Fos as a result of L-AG and/or lactate cannot be distinguished from nuclear immunostaining for c-Fos alone. Therefore, attempts were made to identify the extent of c-Fos induction in phenotypically characterized neurons in efferent targets of the $\mathrm{DMH} / \mathrm{PeF}$ that responded to $\mathrm{L}-\mathrm{AG}$ and/or lactate. This was done using counterstaining for enzymes restricted to neurons (ie TH, ChAT and GAD67) and proteins expressed in the vast majority of neurons of adult rodents (ie NeuN in the PBN (Mullen et al, 1992)). Although identification of c-Fos induction in neurons $v s$ glia could not be conducted in all brain areas in the same group of animals due to limitations of total tissue available, several lines of evidence above demonstrate that at every site we have studied with additional markers, the c-Fos expression has been predominantly seen in the neuronal populations.

Conclusion. The present study demonstrates that selective loss of GABAergic inhibition in critical emotion-regulating regions such as the $\mathrm{DMH} / \mathrm{PeF}$ region results in lactateinduced panic responses similar to those observed in human panic disorder patients. We provide evidence that lactate infusions activate a compromised $\mathrm{DMH} / \mathrm{PeF}$ region that in turn activates forebrain limbic structures, including the BNST, that elicit anxiety-related behavioral responses. In addition, data from the present study are consistent with 
the hypothesis that activation of the $\mathrm{DMH} / \mathrm{PeF}$ region leads secondarily to activation of specific brainstem nuclei to increase anxiety-related behavior and respiration and to densensitize the baroreflex, thus allowing sympathetically mediated tachycardia responses.

\section{ACKNOWLEDGEMENTS}

We gratefully acknowledge Stanley Keim for technical assistance, Pamela E. Kelley for inter-rater analyses and Ryan Wiegand for advice on statistical analyses.

\section{DISCLOSURE/CONFLICT OF INTEREST}

The present work was supported by the National Institute of Mental Health (RO1 MH52619 and RO1 MH065702 to AS); the Wellcome Trust (Research Career Development Fellowship 068558/Z/02/Z to CAL); and the Anxiety Disorders Association of America (Junior Faculty Research Award to PLJ). The authors' have no other disclosures to report.

\section{REFERENCES}

Abshire VM, Hankins KD, Roehr KE, DiMicco JA (1988). Injection of L-allylglycine into the posterior hypothalamus in rats causes decreases in local GABA which correlate with increases in heart rate. Neuropharmacology 27: 1171-1177.

Catelli JM, Sved AF (1988). Enhanced pressor response to GABA in the nucleus tractus solitarii of the spontaneously hypertensive rat. Eur J Pharmacol 151: 243-248.

Chamberlin NL, Saper CB (1994). Topographic organization of respiratory responses to glutamate microstimulation of the parabrachial nucleus in the rat. $J$ Neurosci 14: 6500-6510.

Chan RK, Sawchenko PE (1998). Organization and transmitter specificity of medullary neurons activated by sustained hypertension: implications for understanding baroreceptor reflex circuitry. J Neurosci 18: 371-387.

Chen T, Hui R, Dong YX, Li YQ, Mizuno N (2004). Endomorphin 1- and endomorphin 2-like immunoreactive neurons in the hypothalamus send axons to the parabrachial nucleus in the rat. Neurosci Lett 357: 139-142.

Crestani CC, Alves FH, Resstel LB, Correa FM (2007). Cardiovascular effects of noradrenaline microinjection in the bed nucleus of the stria terminalis of the rat brain. J Neurosci Res 85: 1592-1599.

Dampney RA (1994). Functional organization of central pathways regulating the cardiovascular system. Physiol Rev 74: 323-364.

Davis M, Shi C (1999). The extended amygdala: are the central nucleus of the amygdala and the bed nucleus of the stria terminalis differentially involved in fear versus anxiety? Ann NY Acad Sci 877: 281-291.

Davis SF, Williams KW, Xu W, Glatzer NR, Smith BN (2003). Selective enhancement of synaptic inhibition by hypocretin (orexin) in rat vagal motor neurons: implications for autonomic regulation. J Neurosci 23: 3844-3854.

DiMicco JA, Samuels BC, Zaretskaia MV, Zaretsky DV (2002). The dorsomedial hypothalamus and the response to stress: part renaissance, part revolution. Pharmacol Biochem Behav 71: 469-480.

Ehlers A, Breuer P (1996). How good are patients with panic disorder at perceiving their heartbeats? Biol Psychol 42: 165-182.

Ferguson AV, Samson WK (2003). The orexin/hypocretin system: a critical regulator of neuroendocrine and autonomic function. Front Neuroendocrinol 24: 141-150.
File SE (1980). The use of social interaction as a method for detecting anxiolytic activity of chlordiazepoxide-like drugs. J Neurosci Methods 2: 219-238.

Fitz SD, Keim SR, Sajdyk TJ, Shekhar A (2003). Reboxetine, a potent norepinephrine reuptake inhibitor blocks lactate- and $\mathrm{CO} 2$-induced panic-like responses in panic-prone rats. Soc Neurosci 959: 13.

Fong AY, Stornetta RL, Foley CM, Potts JT (2005). Immunohistochemical localization of GAD67-expressing neurons and processes in the rat brainstem: subregional distribution in the nucleus tractus solitarius. J Comp Neurol 493: 274-290.

Fontes MA, Tagawa T, Polson JW, Cavanagh SJ, Dampney RA (2001). Descending pathways mediating cardiovascular response from dorsomedial hypothalamic nucleus. Am J Physiol Heart Circ Physiol 280: H2891-H2901.

Friedman BH, Thayer JF (1998). Autonomic balance revisited: panic anxiety and heart rate variability. J Psychosom Res 44: 133-151.

Goddard AW, Mason GF, Almai A, Rothman DL, Behar KL, Petroff OA et al (2001). Reductions in occipital cortex GABA levels in panic disorder detected with 1h-magnetic resonance spectroscopy. Arch Gen Psychiatry 58: 556-561.

Gorman JM, Levy GF, Liebowitz MR, McGrath P, Appleby IL, Dillon DJ et al (1983). Effect of acute beta-adrenergic blockade on lactate-induced panic. Arch Gen Psychiatry 40: 1079-1082.

Gorman JM, Papp LA, Coplan JD, Martinez JM, Lennon S, Goetz RR et al (1994). Anxiogenic effects of CO2 and hyperventilation in patients with panic disorder. Am J Psychiatry 151: 547-553.

Hamada T, Koshino Y, Misawa T, Isaki K, Gejyo F (1998). Mitral valve prolapse and autonomic function in panic disorder. Acta Psychiatr Scand 97: 139-143.

Herman JP, Cullinan WE (1997). Neurocircuitry of stress: central control of the hypothalamo-pituitary-adrenocortical axis. Trends Neurosci 20: 78-84.

Herman JP, Prewitt CM, Cullinan WE (1996). Neuronal circuit regulation of the hypothalamo-pituitary-adrenocortical stress axis. Crit Rev Neurobiol 10: 371-394.

Hettema JM, Prescott CA, Myers JM, Neale MC, Kendler KS (2005). The structure of genetic and environmental risk factors for anxiety disorders in men and women. Arch Gen Psychiatry 62 182-189.

Hoehn-Saric R, McLeod DR, Funderburk F, Kowalski P (2004). Somatic symptoms and physiologic responses in generalized anxiety disorder and panic disorder: an ambulatory monitor study. Arch Gen Psychiatry 61: 913-921.

Hosoya Y, Ito R, Kohno K (1987). The topographical organization of neurons in the dorsal hypothalamic area that project to the spinal cord or to the nucleus raphe pallidus in the rat. Exp Brain Res 66: 500-506.

Johnson AK, Gross PM (1993). Sensory circumventricular organs and brain homeostatic pathways. FASEB J 7: 678-686.

Johnson PL, Hollis JH, Moratalla R, Lightman SL, Lowry CA (2005). Acute hypercarbic gas exposure reveals functionally distinct subpopulations of serotonergic neurons in rats. J Psychopharmacol 19: 327-341.

Johnson PL, Shekhar A (2006). Panic-prone state induced in rats with GABA dysfunction in the dorsomedial hypothalamus is mediated by NMDA receptors. J Neurosci 26: 7093-7104.

Jordan D, Mifflin SW, Spyer KM (1988). Hypothalamic inhibition of neurones in the nucleus tractus solitarius of the cat is GABA mediated. J Physiol 399: 389-404.

Kovacs KJ (1998). c-Fos as a transcription factor: a stressful (re)view from a functional map. Neurochem Int 33: 287-297.

Liebowitz MR, Gorman JM, Fyer A, Dillon D, Levitt M, Klein DF (1986). Possible mechanisms for lactate's induction of panic. Am J Psychiatry 143: 495-502.

McCraty R, Atkinson M, Tomasino D, Stuppy WP (2001). Analysis of twenty-four hour heart rate variability in patients with panic disorder. Biol Psychol 56: 131-150. 
McDowall LM, Horiuchi J, Killinger S, Dampney RA (2006). Modulation of the baroreceptor reflex by the dorsomedial hypothalamic nucleus and perifornical area. Am J Physiol Regul Integr Comp Physiol 290: R1020-R1026.

Morgan JI, Curran T (1989). Stimulus-transcription coupling in neurons: role of cellular immediate-early genes. Trends Neurosci 12: $459-462$

Mullen RJ, Buck CR, Smith AM (1992). NeuN, a neuronal specific nuclear protein in vertebrates. Development 116: 201-211.

Nesse RM, Cameron OG, Curtis GC, McCann DS, Huber-Smith MJ (1984). Adrenergic function in patients with panic anxiety. Arch Gen Psychiatry 41: 771-776.

Paxinos G, Watson C (1997). The Rat Brain Stereotaxic Coordinates. Academic Press: San Diego.

Peterson RG (1985). Design and Analysis of Experiments. Marcel Dekker Inc.: New York.

Peyron C, Tighe DK, van den Pol AN, de Lecea L, Heller HC, Sutcliffe JG et al (1998). Neurons containing hypocretin (orexin) project to multiple neuronal systems. J Neurosci 18: 9996-10015.

Pohl R, Balon R, Bechou R, Lycaki H (1994). Lactate-induced anxiety after imapramine and diazepam treatment. Anxiety 1: $54-63$.

Pollock RA, Carter AS, Amir N, Marks LE (2006). Anxiety sensitivity and auditory perception of heartbeat. Behav Res Ther 44: 1739-1756.

Potts JT, Paton JF, Mitchell JH, Garry MG, Kline G, Anguelov PT et al (2003). Contraction-sensitive skeletal muscle afferents inhibit arterial baroreceptor signalling in the nucleus of the solitary tract: role of intrinsic GABA interneurons. Neuroscience 119: 201-214.

Sajdyk TJ, Johnson PL, Fitz SD, Shekhar A (2007). Chronic inhibition of GABA synthesis in the Bed Nucleus of the Stria Terminalis elicits anxiety-like behavior. J Psychopharmacol, in press.

Sakurai T (2007). The neural circuit of orexin (hypocretin): maintaining sleep and wakefulness. Nat Rev Neurosci 8: 171-181.

Samuels BC, Zaretsky DV, DiMicco JA (2004). Dorsomedial hypothalamic sites where disinhibition evokes tachycardia correlate with location of raphe-projecting neurons. Am J Physiol Regul Integr Comp Physiol 287: R472-R478.

Saper CB, Loewy AD, Swanson LW, Cowan WM (1976). Direct hypothalamo-autonomic connections. Brain Res 117: 305-312.

Seifert G, Rehn L, Weber M, Steinhauser C (1997). AMPA receptor subunits expressed by single astrocytes in the juvenile mouse hippocampus. Brain Res Mol Brain Res 47: 286-294.

Senba E, Ueyama T (1997). Stress-induced expression of immediate early genes in the brain and peripheral organs of the rat. Neurosci Res 29: 183-207.

Sevoz-Couche C, Comet MA, Hamon M, Laguzzi R (2003). Role of nucleus tractus solitarius 5-HT3 receptors in the defense reaction-induced inhibition of the aortic baroreflex in rats. J Neurophysiol 90: 2521-2530.

Shekhar A (1993). GABA receptors in the region of the dorsomedial hypothalamus of rats regulate anxiety in the elevated plus-maze test. I. Behavioral measures. Brain Res 627: 9-16.

Shekhar A, DiMicco JA (1987). Defense reaction elicited by injection of GABA antagonists and synthesis inhibitors into the posterior hypothalamus in rats. Neuropharmacology 26: 407-417.
Shekhar A, Hingtgen JN, DiMicco JA (1987). Selective enhancement of shock avoidance responding elicited by GABA blockade in the posterior hypothalamus of rats. Brain Res 420: 118-128.

Shekhar A, Hingtgen JN, DiMicco JA (1990). GABA receptors in the posterior hypothalamus regulate experimental anxiety in rats. Brain Res 512: 81-88.

Shekhar A, Johnson PL, Sajdyk TJ, Fitz SD, Keim SR, Kelley PE et al (2006). Angiotensin-II is a putative neurotransmitter in lactate-induced panic-like responses in rats with disruption of GABAergic inhibition in the dorsomedial hypothalamus. J Neurosci 26: 9205-9215.

Shekhar A, Katner JS (1995). Dorsomedial hypothalamic GABA regulates anxiety in the social interaction test. Pharmacol Biochem Behav 50: 253-258.

Shekhar A, Keim SR (1997). The circumventricular organs form a potential neural pathway for lactate sensitivity: implications for panic disorder. J Neurosci 17: 9726-9735.

Shekhar A, Keim SR, Simon JR, McBride WJ (1996). Dorsomedial hypothalamic GABA dysfunction produces physiological arousal following sodium lactate infusions. Pharmacol Biochem Behav 55: $249-256$.

Shioiri T, Kojima-Maruyama M, Hosoki T, Kitamura H, Tanaka A, Yoshizawa $\mathrm{M}$ et al (2005). Dysfunctional baroreflex regulation of sympathetic nerve activity in remitted patients with panic disorder. A new methodological approach. Eur Arch Psychiatry Clin Neurosci 255: 293-298.

Singewald N, Salchner P, Sharp T (2003). Induction of c-Fos expression in specific areas of the fear circuitry in rat forebrain by anxiogenic drugs. Biol Psychiatry 53: 275-283.

Singewald N, Sharp T (2000). Neuroanatomical targets of anxiogenic drugs in the hindbrain as revealed by Fos immunocytochemistry. Neuroscience 98: 759-770.

Starcevic V, Uhlenhuth EH, Kellner R, Pathak D (1993a). Comparison of primary and secondary panic disorder: a preliminary report. J Affect Disord 27: 81-86.

Starcevic V, Kellner R, Uhlenhuth EH, Pathak D (1993b). The phenomenology of panic attacks in panic disorder with and without agoraphobia. Compr Psychiatry 34: 36-41.

Stein DJ, Bouwer C (1997). A neuro-evolutionary approach to the anxiety disorders. J Anxiety Disord 11: 409-429.

Street LL, Craske MG, Barlow DH (1989). Sensations, cognitions and the perception of cues associated with expected and unexpected panic attacks. Behav Res Ther 27: 189-198.

The American Psychiatric Association (2000). Diagnostic and Statistical Manual of Mental Disorders-Fourth Edition-Text Revision (DSM-IV-TR). The American Psychiatric Association: Washington, DC.

Thielen SK, Shekhar A (2002). Amygdala priming results in conditioned place avoidance. Pharmacol Biochem Behav 71: 401-406.

Thompson RH, Swanson LW (1998). Organization of inputs to the dorsomedial nucleus of the hypothalamus: a reexamination with Fluorogold and PHAL in the rat. Brain Res Brain Res Rev 27: 89-118.

Vickers K, McNally RJ (2005). Respiratory symptoms and panic in the National Comorbidity Survey: a test of Klein's suffocation false alarm theory. Behav Res Ther 43: 1011-1018.

Winsky-Sommerer R, Yamanaka A, Diano S, Borok E, Roberts AJ, Sakurai $\mathrm{T}$ et al (2004). Interaction between the corticotropinreleasing factor system and hypocretins (orexins): a novel circuit mediating stress response. J Neurosci 24: 11439-11448.

Supplementary Information accompanies the paper on the Neuropsychopharmacology website (http://www.nature.com/npp) 